\title{
Exosomal miRNA-19b-3p of tubular epithelial cells promotes M1 macrophage activation in kidney injury
}

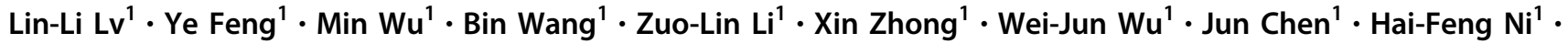 \\ Tao-Tao Tang ${ }^{1} \cdot$ Ri-Ning Tang ${ }^{1} \cdot$ Hui-Yao Lan $\mathbb{D}^{2} \cdot$ Bi-Cheng Liu ${ }^{1}$
}

Received: 6 October 2018 / Revised: 25 April 2019 / Accepted: 3 May 2019 / Published online: 16 May 2019

(c) ADMC Associazione Differenziamento e Morte Cellulare 2019

\begin{abstract}
Tubulointerstitial inflammation is a common characteristic of acute and chronic kidney injury. However, the mechanism by which the initial injury of tubular epithelial cells (TECs) drives interstitial inflammation remains unclear. This paper aims to explore the role of exosomal miRNAs derived from TECs in the development of tubulointerstitial inflammation. Global microRNA(miRNA) expression profiling of renal exosomes was examined in a LPS induced acute kidney injury (AKI) mouse model and miR-19b-3p was identified as the miRNA that was most notably increased in TEC-derived exosomes compared to controls. Similar results were also found in an adriamycin (ADR) induced chronic proteinuric kidney disease model in which exosomal miR-19b-3p was markedly released. Interestingly, once released, TEC-derived exosomal miR-19b-3p was internalized by macrophages, leading to M1 phenotype polarization through targeting NF- $\mathrm{KB} / \mathrm{SOCS}-1$. A dual-luciferase reporter assay confirmed that SOCS-1 was the direct target of miR-19b-3p. Importantly, the pathogenic role of exosomal miR-19b-3p in initiating renal inflammation was revealed by the ability of adoptively transferred of purified TEC-derived exosomes to cause tubulointerstitial inflammation in mice, which was reversed by inhibition of miR-19b-3p. Clinically, high levels of miR-19b-3p were found in urinary exosomes and were correlated with the severity of tubulointerstitial inflammation in patients with diabetic nephropathy. Thus, our studies demonstrated that exosomal miR-19b-3p mediated the communication between injured TECs and macrophages, leading to M1 macrophage activation. The exosome/miR19b-3p/SOCS1 axis played a critical pathologic role in tubulointerstitial inflammation, representing a new therapeutic target for kidney disease.
\end{abstract}

These authors contributed equally: Lin-Li Lv, Ye Feng

Edited by J.M. Hardwick

Supplementary information The online version of this article (https:// doi.org/10.1038/s41418-019-0349-y) contains supplementary material, which is available to authorized users.

Lin-Li Lv

lvlinli@seu.edu.cn

$\triangle$ Bi-Cheng Liu

liubc64@163.com

1 Institute of Nephrology, Zhongda Hospital, Southeast University School of Medicine, Nanjing, Jiangsu Province 210009, China

2 Department of Medicine and Therapeutics, Li Ka Shing Institute of Health Sciences, and Liu Che Woo Institute of Innovative Medicine, Chinese University of Hong Kong, Hong Kong, SAR 999077, China

\section{Introduction}

Tubulointerstitial inflammation is a common characteristic of many types of acute and chronic kidney injury. Thus, understanding the mechanisms of tubulointerstitial inflammation is a critical step towards the development of effective therapies for kidney disease. Tubular epithelial cells (TECs) are the most populous cell type in the kidney and exert diverse regulatory functions in both normal and pathological conditions. TECs are usually the initial site of injuries caused by hypoxia, proteinuria, toxins, metabolic disorders, and senescence. Accumulating evidence has demonstrated that the renal tubules are not only a victim of injury but also a driving force in the progression of kidney diseases [1-3]. However, the mechanisms by which the initial tubular injury triggers kidney inflammation and fibrosis remain unclear. 
Table 1 Primers for quantitative RT-PCR

\begin{tabular}{lll}
\hline Primer & Forward $\left(5^{\prime}-3^{\prime}\right)$ & Reverse $\left(5^{\prime}-3^{\prime}\right)$ \\
\hline Mouse IL-1 $\beta$ & TGCCACCTTTTGACAGTGATG & AAGGTCCACGGGAAAGACAC \\
Mouse IL-6 & AAAGAGTTGTGCAATGGCAATTCT & AAGTGCATCATCGTTGTTCATACA \\
Mouse TNF- $\alpha$ & CATCTTCTCAAAATTCGAGTGACAA & TGGGAGTAGACAAGGTACAACCC \\
Mouse GAPDH & GCATGGCCTTCCGTGTTC & GATGTCATCATACTTGGCAGGTTT \\
Mouse MCP-1 & CTTCTGGGCCTGCTGTTCA & CCAGCCTACTCATTGGGATCA \\
Mouse SOCS-1 & CACTCACTTCCGCACCTTCC & CAGCCGGTCAGATCTGGAAG \\
Mouse iNOS & CAGATCGAGCCCTGGAAGAC & CTGGTCCATGCAGACAACCT \\
\hline
\end{tabular}

Recent studies revealed that the crosstalk between the tubular epithelium and the interstitial cells might play important role in both repair/regeneration and disease progression [4]. TECs are a rich source of chemokines and cytokines in response to injury, which in turn promote renal injury by recruiting inflammatory cells into the injured kidneys. Macrophages are the predominant immune cells that mediate the inflammatory process [5]. However, the mechanisms by which TEC-derived inflammatory signals spread to macrophages during the development of interstitial inflammation remain largely unknown.

Exosomes are extracellular vesicles released by all cell types and are initially considered to be cellular garbage [6-8]. Over the past decades, numerous studies have determined that exosomes mediate intracellular communication by transferring cell-specific cargo, including proteins, lipids and genetic information, to recipient cells [9]. Our previous studies revealed that exosomal transfer of CCL2 mRNA from TECs to macrophages constitutes a critical mechanism of albumin-induced tubulointerstitial inflammation [10]. MicroRNAs(miRNAs) are a class of small noncoding RNAs that modulate gene expression via binding target mRNAs for degradation or translational repression. Recent studies found that miRNAs are commonly enriched in exosomes and are merging as crucial regulators of cellular function through vesicle mediated cellular communication $[11,12]$. miR-486-5p movement from endothelial colony forming cells to endothelial cells through exosomes could protect against endothelial injury [13]. Immune cells could also take up exosomal miRNAs to regulate the inflammatory responses as previously reported [14]. Increasing evidence has also demonstrated the crucial role of miRNAs in the development of kidney disease and inflammatory disease [15, 16, 17]. However, the role of exosomal miRNAs in the development of tubulointerstitial inflammation remains largely unclear. In the study, we posited that exosomal miRNA released by TECs could initiate tubulointerstitial inflammation through conveying the injury signals to activate macrophages.

\section{Materials and methods}

\section{Animal models of acute and chronic kidney injury}

All the mice were purchased from the Beijing Vital River Laboratory Animal Technology Co., Ltd. and were studied with protocols approved by the Ethics Committee of Southeast University. For acute kidney injury (AKI) model, 6-8-week-old male BALB/c mice ( $n=6$ per group,) were administered intraperitoneally with either LPS (L2630, Sigma Aldrich, USA) at a dose of $10 \mathrm{mg} / \mathrm{kg}$ body weight dissolved in $200 \mu \mathrm{l}$ PBS or control PBS randomly as reported before [10]. At $24 \mathrm{~h}$ after LPS or PBS injection, mice were euthanized and blood and urine were collected for serum creatinine and urinary protein excretion detection. Renal cortex was harvested and processed for further analysis. For miRNA array screening, exosome samples were purified from kidney of mice with LPS treatment and controls ( $n=4$ per group). For chronic proteinuric kidney disease model, male $\mathrm{C} 57 \mathrm{BL} / 6 \mathrm{~J}$ mice ( $n=6$ per group) received a single intravenous injection of either $18 \mathrm{mg} / \mathrm{kg}$ body weight adriamycin (ADR, D1515, Sigma Aldrich, USA) dissolved in $\mathrm{ddH}_{2} \mathrm{O}$ or an equal volume of $\mathrm{ddH}_{2} \mathrm{O}$ randomly. Urine was collected $0,7,14$, and 21 days after injection of $\mathrm{ADR}$ or $\mathrm{ddH}_{2} \mathrm{O}$. At 23 days after injection, blood and renal cortex were collected from both groups of mice. To specifically identify the role of exosomal miR$19 \mathrm{~b}-3 \mathrm{p}$ in kidney injury, 8-week-old male BALB/c mice ( $n$ $=6$ per group) were randomly intrarenally injected with exosomes generated in vitro by $8 \times 10^{6}$ TECs that were transfected with miR-19b inhibitor or negative control under high BSA conditions $(20 \mathrm{mg} / \mathrm{ml}$, Sigma Aldrich, USA). Mice were sacrificed $24 \mathrm{~h}$ after exosome injection.

\section{Cell culture}

An immortalized mouse tubular epithelial cells (mTECs) (a gift from Dr. Jeffery B. Kopp, National Institutes of Health) were maintained in DMEM-F12(Hyclone, USA) supplemented with $1 \%(\mathrm{v} / \mathrm{v})$ penicillin streptomycin $(\mathrm{P} / \mathrm{S}$, Gibco, USA) and 10\% fetal bovine serum (FBS, Gibco, 

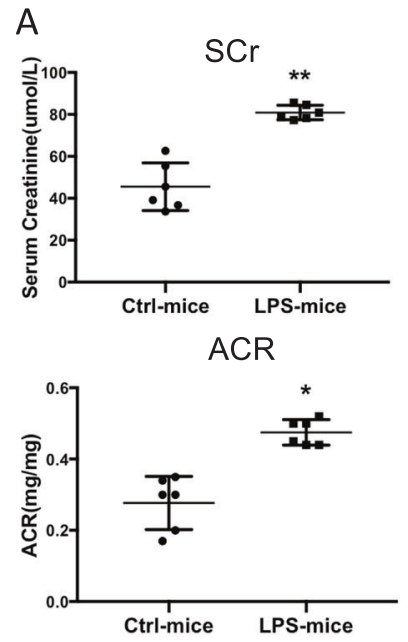

C Exosomes purified from kidney (TEM)

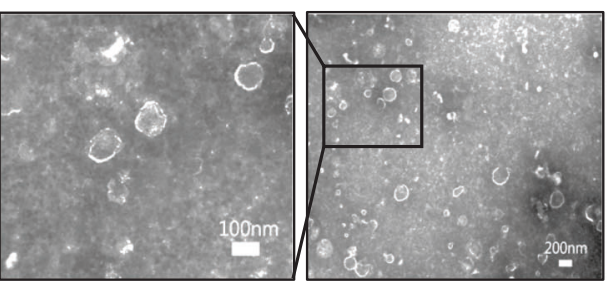

Ctrl
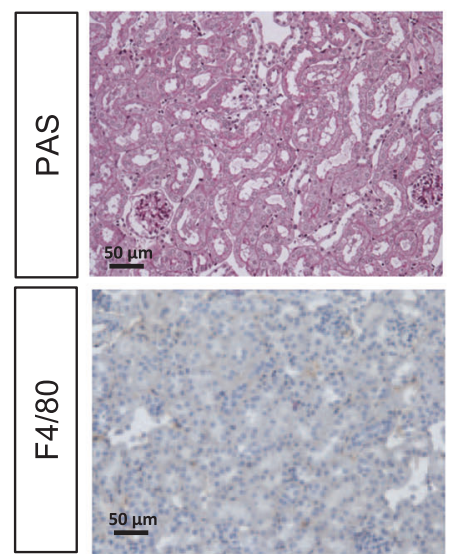

LPS

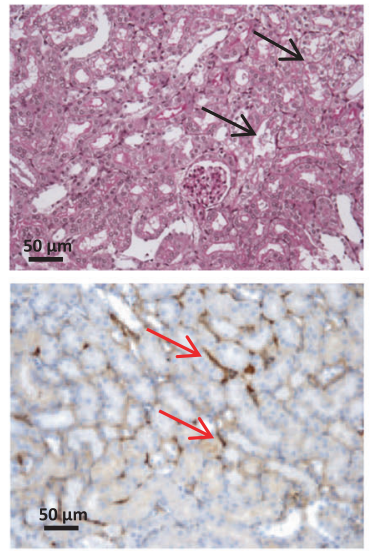

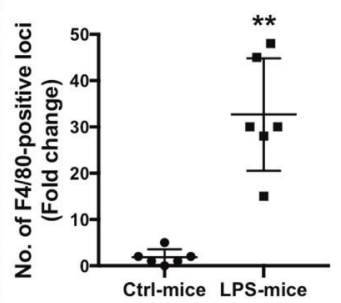
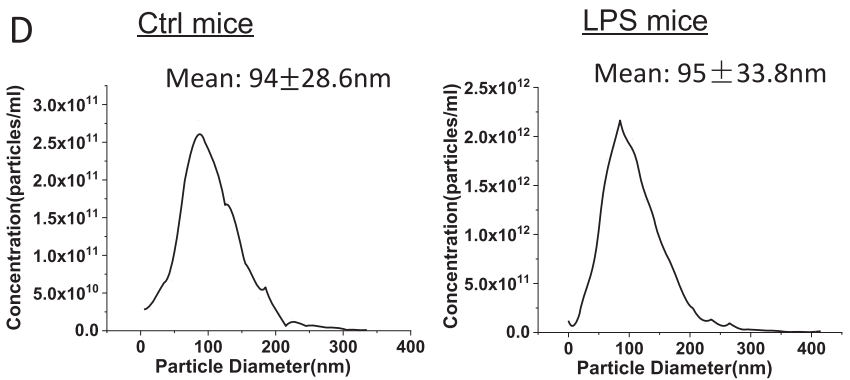

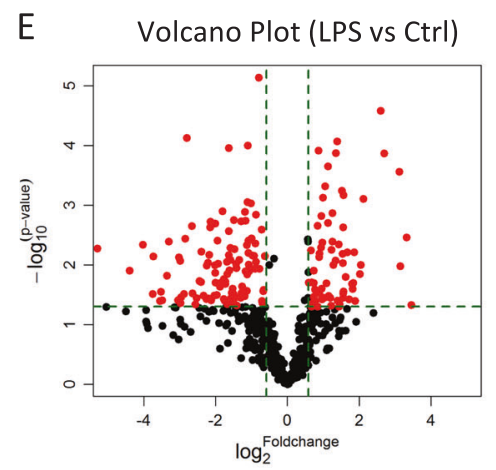

G kidney-exo miR-19b-3p

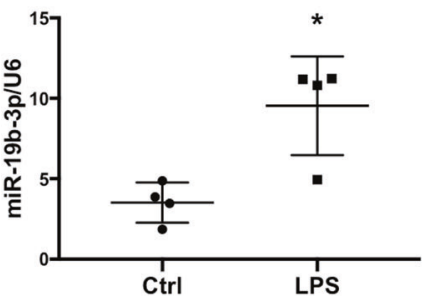

USA). Raw264.7 macrophages were cultured in RPMI 1640 (Hyclone, USA) media supplemented with $1 \%(\mathrm{v} / \mathrm{v})$ penicillin streptomycin (P/S, Gibco) and 10\% FBS (0500, Sciencell, USA). Bone-marrow derived macrophages (BMMs) were harvested from femurs of wild type C57BL/ $6 \mathrm{~J}$ mice and differentiated into macrophages using DMEM/ F12 supplemented with $10 \%(\mathrm{v} / \mathrm{v})$ FBS, $1 \%(\mathrm{v} / \mathrm{v}) \mathrm{P} / \mathrm{S}$,
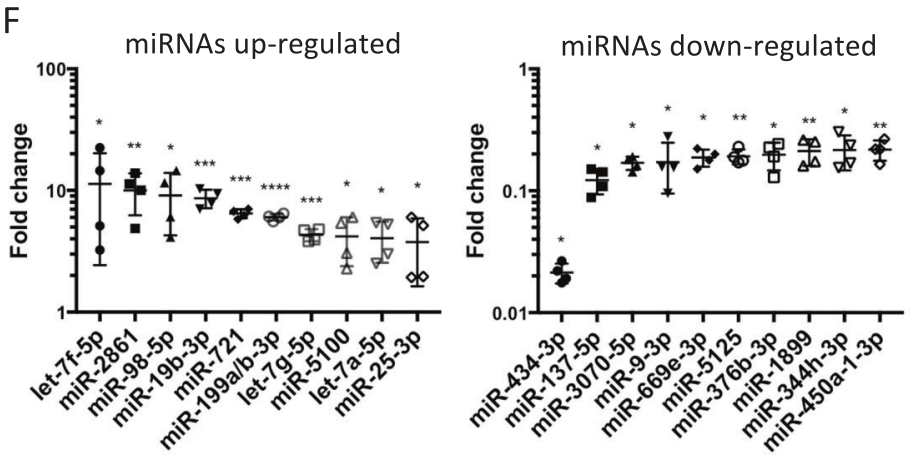

H Tubular-exo miR-19b-3p

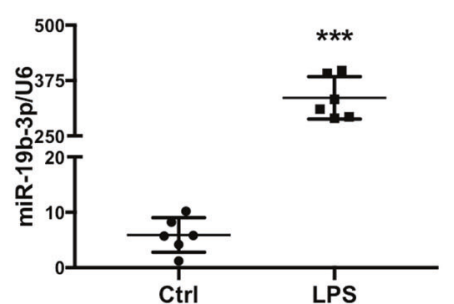

$40 \mathrm{ng} / \mathrm{ml}$ mouse recombined M-CSF (416-ML, R\&D System, USA) for 7 days.

\section{Isolation and characterization of exosomes}

For kidney exosome isolation, $100 \mathrm{mg}$ renal cortex was harvested and digested with collagenase and trypsin for $2 \mathrm{~h}$ 
Fig. 1 MiRNA profile of renal exosomes in LPS-induced AKI model. a Serum levels of creatinine (Scr, $* * p=0.0015$ vs Ctrl-mice) and urine albumin-to-creatinine ratios (ACR, ${ }^{*} p=0.0249$ vs Ctrl-mice) in LPS-induced acute kidney injury model at $24 \mathrm{~h}$. b Histologic and immunohistologic changes in LPS-induced mice, with PAS staining and F4/80 immunostaining, respectively. Tubular injury (black arrow) and $\mathrm{F} 4 / 80$ positive macrophages in tubulointerstitium were indicated (red arrow). The fold change of the numbers of F4/80 positive loci between two groups were calculated. $* * p=0.0073$ vs Ctrl-mice. Scale bar, $50 \mu \mathrm{m}$. c Representative electron micrograph of exosomes purified from the kidneys. Scale bars, 200 and $100 \mathrm{~nm}$. d The size of renal exosomes analyzed by NTA. The mean vesicle diameter was $94 \pm$ $28.6 \mathrm{~nm}$ and $95 \pm 33.8 \mathrm{~nm}$ for control and LPS mice. e The volcano plot of miRNAs analyzed by miRCURY LNA Arrays (Exiqon, Denmark) from kidney exosomes. The volcano plot was constructed with $\log _{2}$ expression of fold change and the corresponding tempered $\log _{2} \mathrm{p}$ value of all miRNAs. The vertical lines correspond to 2.0 -fold up and down, respectively, and the horizontal line represents a $p$-value of 0.05. f Top ten miRNAs increased or decreased in renal exosomes from LPS-treated mice vs control mice. Data show fold change in kidney-exosomes of LPS-mice vs of Ctrl-mice. $\mathbf{g}$ Expression of miR$19 b-3 p$ in the renal exosomes of LPS-injected or control mice validated by real time RT-PCR ( ${ }^{*} p=0.0461$ vs Ctrl-mice). h Expression of miR-19b-3p in the tubule-derived exosomes from LPS-injected or control mice $(* * * p=0.0004$ vs Ctrl-mice). Data presented as mean \pm $\mathrm{SD}$ or mean \pm S.E.M. of individuals included in each group. $\mathrm{P}$ values were calculated using unpaired Student's $t$-test. ( $n=6$ for each group of mice. In the miRNA array analysis, exosome samples from four mice for each group were included for the screening.). Exo exosomes

at $37^{\circ} \mathrm{C}$. Then the renal homogenate was subjected for exosome extraction as reported before [10]. To isolate tubular exosomes, tubular fragments were isolated by 80 -mesh stainless sifter, followed by exosome purification with ultracentrifugation [18]. For in vitro experiments, TECs were cultured in the presence or absence of $20 \mathrm{mg} / \mathrm{ml} \mathrm{BSA}$ in serum-free DMEM/F12 medium for $24 \mathrm{~h}$. And then cells were cultured $24 \mathrm{~h}$ in medium without serum and BSA for exosome purification. Urinary samples were collected from diabetic nephropathy (DN) and type 2 diabetes mellitus (T2DM) patients for exosome purification using differential ultracentrifugation as described previously [10]. All patients were given the written informed consent and the study was approved by The Ethics Committee of Zhongda Hospital. Briefly, the samples were centrifuged at $2000 \times \mathrm{g}$ and $13,500 \times g$ for $20 \mathrm{~min}$ to eliminate the cell debris and large vesicles, respectively. The pellet was washed once with PBS and purified by centrifugation at $200,000 \times g$ for $2 \mathrm{~h}$ (Type 70 Ti rotor, Beckman Coulter Optima L-80 XP).

\section{Transmission electron microscope (TEM)}

The exosome samples were diluted 5 times with PBS and then applied to 200-mesh nickel grids. Samples were stained with $2 \%$ phosphotungstic acid for $5 \mathrm{~min}$ at room temperature, and air-dried. Exosomes were detected through transmission electron microscope (Hitachi HT 7700, Japan) at $80 \mathrm{kV}$.

\section{ZetaView nanoparticle tracking analysis (NTA)}

NTA was performed with the ZetaView PMX 110 (Particle Metrix, Meerbusch, Germany). Purified exosomes were diluted with PBS buffer to measure the particle size and concentration. The corresponding software, ZetaView 8.04.02, was used to analyze the data. To convert the yield from concentration to an accurate number of particles, dilution factors and resuspension volumes were used.

\section{miRNA expression profiling}

The renal exosomes isolated from control $(n=4)$ and LPStreated $(n=4)$ mice were lysed and total RNAs were extracted using miRNeasy Micro Kit (217084, QIAGEN, Germany) according to the manufacture's protocol. We performed miRNA expression profiling using the miRCURY LNA Array (version 11.0, Exiqon, Denmark) system. RNA labeling and array hybridization were performed according to Exiqon's manual and analyzed with the Axon GenePix 4000B microarray scanner. Significance of differentially-expressed miRNAs between two groups were identified through fold change and $P$-value. Hierarchical clustering was performed to show distinguishable miRNA expression profiling among samples.

\section{Cell transfection with miRNA mimics and inhibitors}

Both Raw 264.7 and bone marrow derived macrophages (BMMs) were transfected with control mimic or miR-19b$3 \mathrm{p}$ mimic (GenePharma, China), negative control (NC) inhibitor or miR-19b-3p inhibitor (GenePharma, China) at a concentration of $100 \mathrm{nM}$ using Lipofectamine 2000 (11668019, Invitrogen, USA) as instructed by the manufacturer's protocols. miR-19b-3p mimic, and miR-19b-3p inhibitor sequences are as follows:

miR-19b-3p mimic (5'-UGUGCAAAUCCAUGCAAAA CUGA-3'

3'-AGUUUUGCAUGGAUUUGCACAUU-5')

miR-19b-3p inhibitor (5'-UCAGUUUUGCAUGGAUU UGCACA-3”)

\section{Luciferase reporter assay}

$293 \mathrm{~T}$ cells $\left(10^{5}\right)$ were transfected with $3^{\prime}$ UTR luciferase reporter constructs (3'UTR-NC, 3'UTR-SOCS1, 3'UTRSOCS1-mutant), miRNA (miRNA-NC or miR-19b-3p) and Renilla luciferase using Lipofectamine 2000, according to manufacturer's instructions (Genechem, Shanghai, China). After $48 \mathrm{~h}$ of transfection, luciferase activity of cells was measured using a Dual Luciferase Assay Kit (Promega, E1910) and microplate reader (BioTeK, H1). Luciferase activity of each groups was calculated and graphed. Renilla 
A

ADR $(18 \mathrm{mg} / \mathrm{kg})$

tail vein

Sacrificed

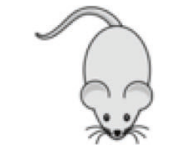

6-8 weeks old

C57BL/6J mice
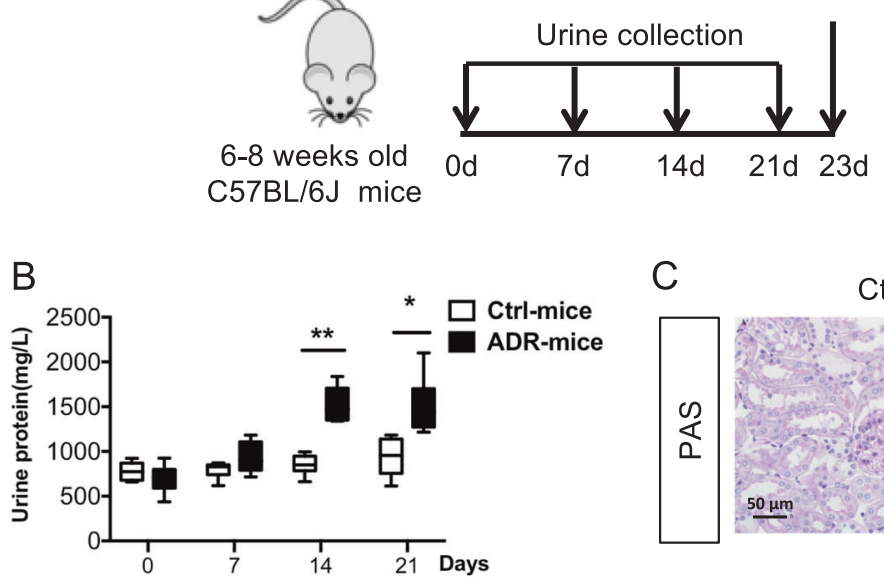

C

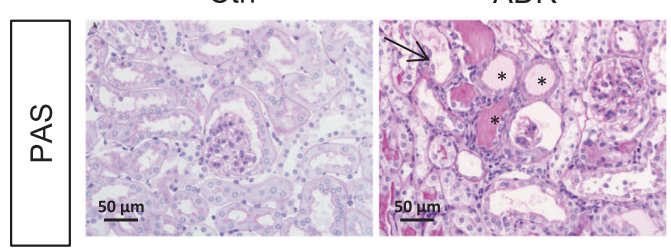

D
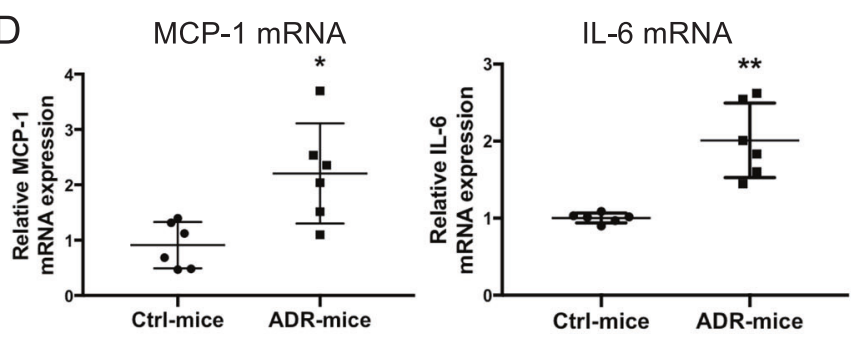

E

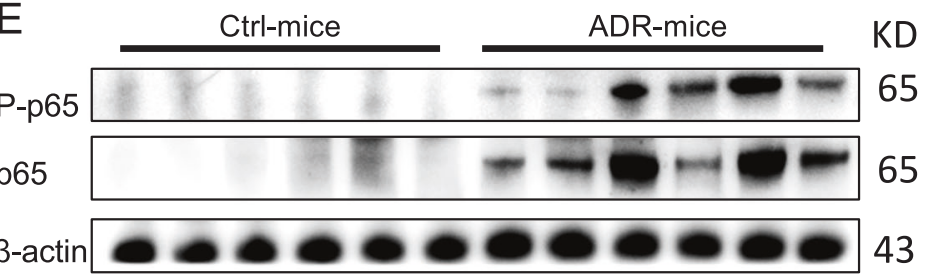

$\beta$-actin

F

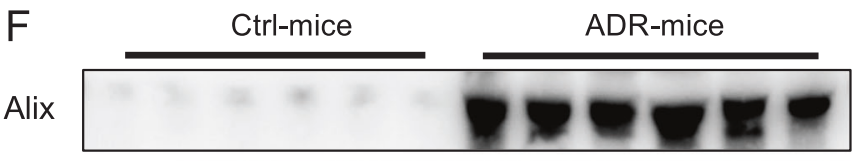

CD9

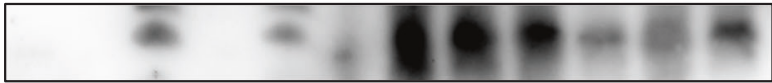

CD63

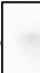

KD

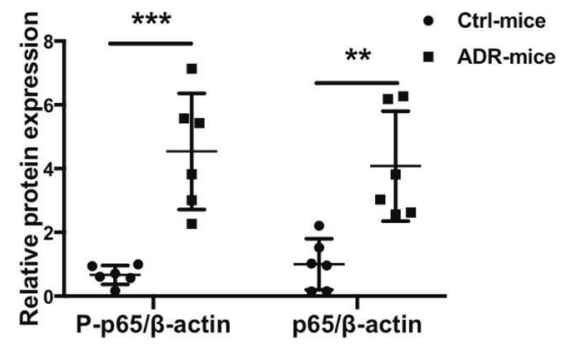

25

36

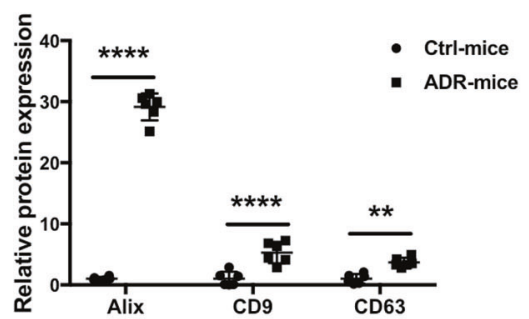

G Kidney-exo miR-19b-3p

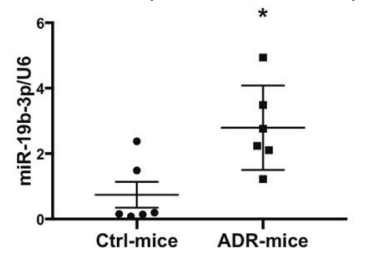


Fig. 2 miR-19b-3p increased in kidney exosomes in adriamycininduced proteinuric disease model. a A schematic review of in vivo experiment. b Proteinuria in mice at $0,7,14$ and 21 days. Adriamycin (ADR) mice developed severe proteinuria 2 weeks after injection. $(* * p=0.0012$ for 14 days and $* p=0.01$ for 21 days vs Ctrl-mice) c Histologic (PAS staining) changes at 23 days after injection of ADR or PBS. ADR induced significant tubular injury and protein cast, which were pointed by arrows and stars. Scale bar, $50 \mu \mathrm{m}$. d mRNA expression of inflammatory cytokines in the kidney by real-time PCR. ADR mice showed significant upregulation of MCP-1 $\left({ }^{*} p=0.0267\right.$ vs Ctrl-mice), IL-6 (**p $=0.0033$ vs Ctrl-mice) in the kidney. e Representative western blotting results from three independent experiments and quantification of P-p65(*** $p=0.0002$ vs Ctrl-mice), p65(** $p=$ 0.0019 vs Ctrl-mice). f Representative western blotting and quantification of exosomal markers (Alix, CD9 and CD63) in renal exosomes $(* * * p<0.0001, * * p=0.0046$ vs Ctrl-mice). g Expression of miR$19 \mathrm{~b}-3 \mathrm{p}$ in the renal exosomes of ADR-injected or control mice ${ }^{*} p=$ 0.0463 vs Ctrl-mice). Data presented as mean \pm S.E.M. of individuals included in each group. $p$ values were calculated using unpaired Student's $t$-test ( $n=6$ for each group of mice). Exo exosomes, Ctrl control

luciferase was used to normalize the value of firefly luciferase.

\section{Isolation and tracking of labeled exosomes}

TECs were cultured in serum free medium for $24 \mathrm{~h}$. Then cells were stained by $\mathrm{DiO}$ dye $(5 \mathrm{mg} / \mathrm{ml}$, Beyotime, China) for $30 \mathrm{~min}$ at $37^{\circ} \mathrm{C}$ and the free dye was washed away with PBS. Labeled exosomes were isolated from cell supernatants using ultracentrifugation and applied to the Raw264.7 macrophages that were grown in exosome-free medium. After $24 \mathrm{~h}$, uptake of labeled exosomes was visualized by the confocal microscope Fluoview FV1000 Version2.1 (Olympus) using a $488 \mathrm{~nm}$ laser.

\section{Co-culture experiments}

To mimic the interaction between TEC-derived exosomes and macrophages, Transwell Permeable Support systems (Corning) for 12-well plates with a $0.4 \mu \mathrm{m}$ pore-size filter were used as the manufacturer's protocols. Donor TECs were plated in upper chamber followed by DiO-labeling. Medium were removed and replaced by serum-free DMEM/ F12, while recipient macrophages were then seeded in 12well plates without serum. All co-cultured experiments were conducted in the presence or absence of $20 \mathrm{mg} / \mathrm{ml} \mathrm{BSA} \mathrm{for}$ $24 \mathrm{~h}$. Uptake of DiO-labeled exosomes was visualized by the confocal microscope Fluoview FV1000 Version2.1 (Olympus) using a $488 \mathrm{~nm}$ laser.

\section{RNA isolation and detection of mRNA and miRNA}

Total RNA isolation was performed using TRIzol following the manufacturer's instruction (TAKARA, Japan). miRNAs were reverse-transcribed and detected with All-in-One miRNA First-Strand cDNA Synthesis Kit and All-in-One miRNA qPCR Kit (QP015, GeneCopoeia, USA), U6 snRNA was used as internal control. Primers of miR19b-3p and U6 were purchased from GeneCopoeia. mRNA was reverse transcribed using PrimeScript RT reagent Kit (TAKARA) and PCR was conducted using SYBR Premix Ex Taq and 7300 Real-Time PCR System (Applied Biosystems). The data was normalized to the expression of GAPDH. Primer sequences are shown in Table 1.

\section{Western blotting analysis}

Cells, renal tissues and purified exosomes were lysed in icecold RIPA Lysis buffer (89901, Thermo Scientific, USA) supplemented with protease inhibitor cocktail and protein concentration was determined by BCA assay (KeyGEN, BioTECH). Proteins were subjected to $10 \%$ SDS-PAGE (Thermo Scientific) and transferred to PVDF membranes (Millipore). The membranes were blocked with $5 \% \mathrm{w} / \mathrm{v}$ BSA (Biosharp) in TBST and incubated overnight with primary antibodies as followed: anti-Alix (1:500, sc-53540, Santa Cruz Biotechnology), anti-CD9 (1:2000, ab92726, Abcam), anti-CD63 (1:2000, ab213090, Abcam), antiSOCS-1 (1:1000, ab62584, Abcam), anti-P-p65 (1:1000, 3033S, Cell Signaling Technology), anti-p65 (1:2000, 8242S, Cell Signaling Technology), anti- $\beta$-actin (1:5000, sc-47778, Santa Cruz Biotechnology), anti-iNOS (1:2000, ab15323, Abcam). Goat anti-mouse or anti-rabbit secondary antibodies (Cell Signaling Technology) were used for detection on ImageQuant LAS 500(GE Healthcare).

\section{Immunofluorescence}

Immunofluorescence microscopy was used to identify the subcellular localization of SOCS-1 in the renal cortex from DN patients with renal biopsies. The slides of kidney were incubated with primary antibody against SOCS-1(1:200, ab62584, Abcam) followed by incubation with FITC-tagged secondary antibodies (Bioss, bs-0295D, Beijing, China). All samples were treated with DAPI dye for nuclear staining and detected by the confocal microscope Fluoview FV1000 Version2.1 (Olympus).

\section{Statistical analysis}

Data are expressed as mean \pm standard deviation (SD) or as mean \pm standard error of mean (S.E.M) of each group. A two-tailed unpaired Student's $t$-test was used for comparison between two groups, and one-way ANOVA were performed for comparisons of data with more than two groups followed by Bornferroni correction for multiple 


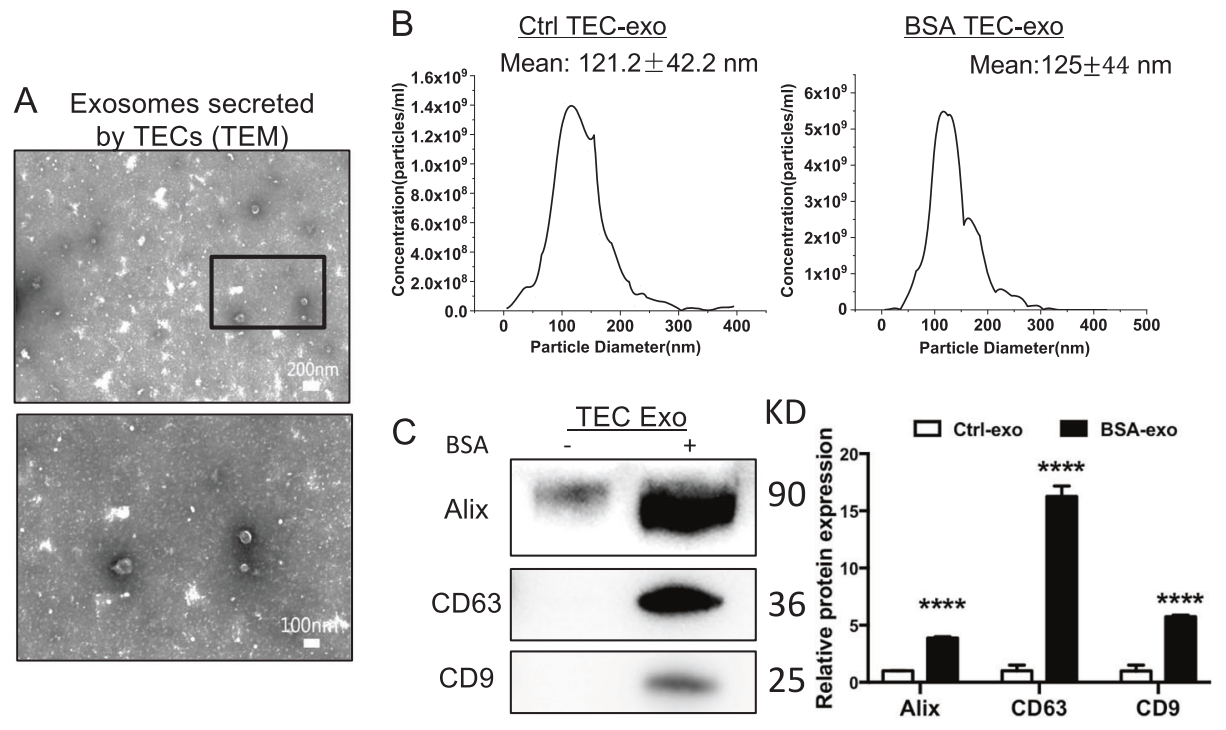

D TEC-RAW264.7 co-culture

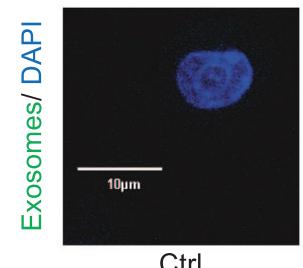

Ctrl

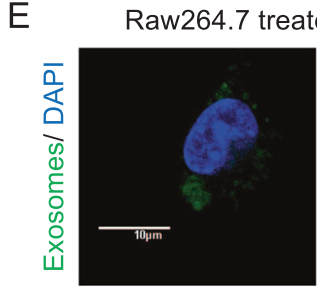

Ctrl-exo(DIO)

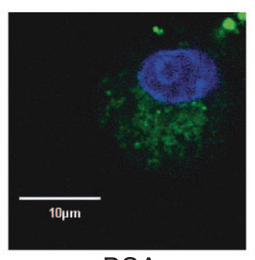

BSA
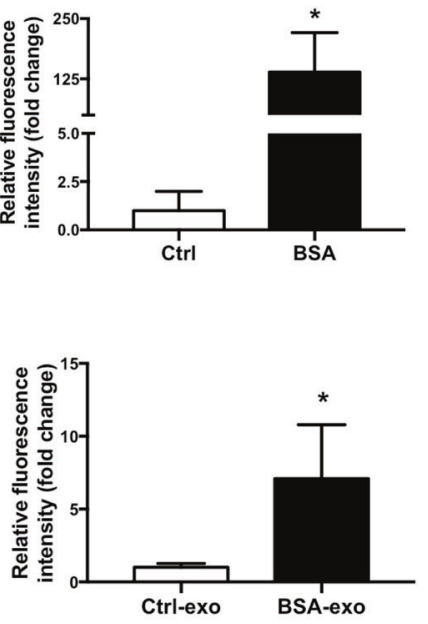

BSA-exo(DIO)

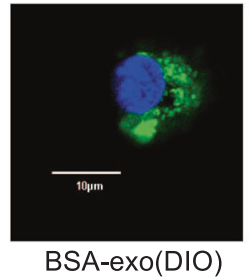

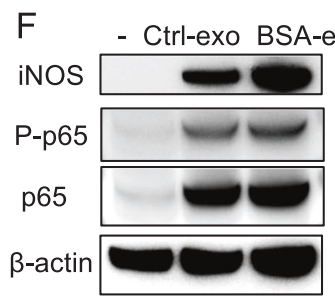
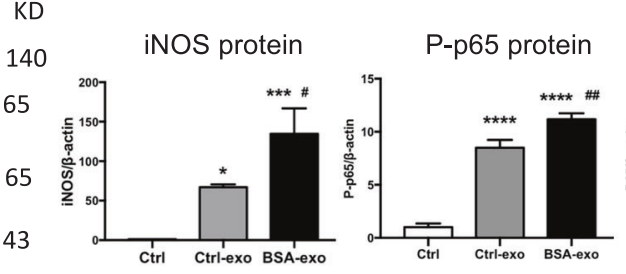

p65 protein
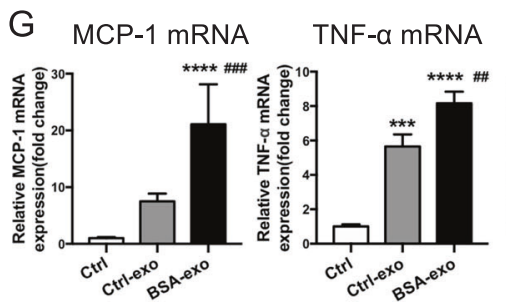

IL-1 $\beta$ mRNA

IL-6 mRNA
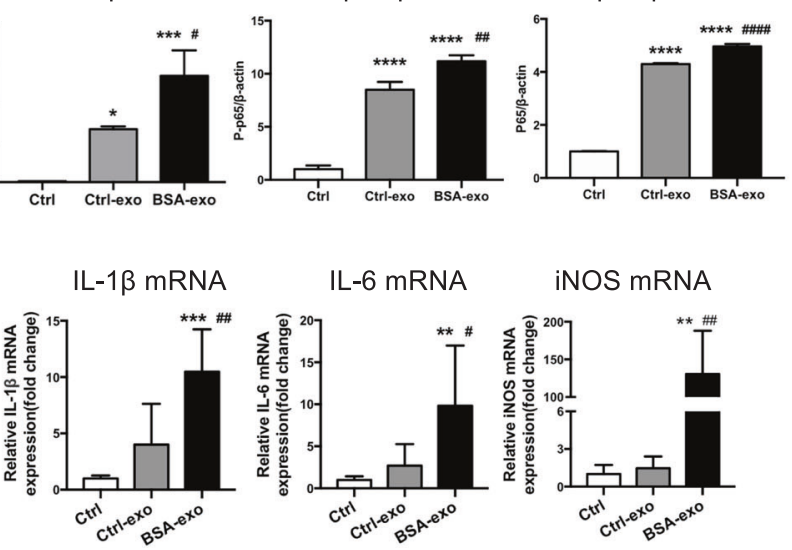
Fig. 3 Increasing internalization of TEC-derived exosomes promoted M1 macrophage activation. a Representative electron micrograph images of exosomes isolated from TEC conditioned medium. Scale bar, 200 and $100 \mathrm{~nm}$. b Shown are size distribution of exosomes isolated from TECs conditioned medium by nanoparticle analysis (NTA). c Representative western blotting and quantification of exosomal markers (including Alix, CD9 and CD63) in TEC-derived exosomes with or without BSA $(* * * * p<0.0001$ vs exosomes from control TECs). d DiO-labeled TECs were co- cultured with recipient Raw264.7 macrophages using transwell system in the absence/presence of BSA for $24 \mathbf{h}$ Relative fluorescence intensity was quantified to identify the uptake of exosomes. ( $* p=0.012$ vs Ctrl). Scale bar, 10 $\mu \mathrm{m}$. e Exosomes were purified from DiO-labeled TECs and were then applied to recipient Raw264.7 macrophages. Relative fluorescence intensity was calculated ( ${ }^{*} p=0.0461$ vs Ctrl-exosomes). f Representative western blotting and quantification of iNOS, P-p65, p65 in recipient Raw264.7 macrophages treated with TEC-derived exosomes in the absence or presence of BSA. $\mathbf{g}$ Inflammatory cytokine mRNA (MCP-1, IL1 $\beta$, TNF- $\alpha$, IL6) and iNOS mRNA expression in recipient RAW264.7 macrophages were detected by real-time PCR. ** $p<0.01$; $* * * p<0.001 ; * * * * p<0.0001$ compared with cells without exosomes;

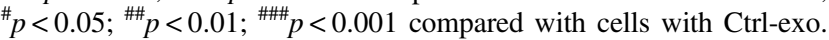
Ctrl exo exosomes from TECs without BSA, $p$ values were calculated using one-way ANOVA test. Bornferroni-corrected $\alpha$ threshold was used for statistical significance of multiple comparison. BSA-exo exosomes from TECs with BSA treatment. Data presented as mean \pm S.E.M. of three independent experiments

comparisons. All analyses were carried out by GraphPad Prism 5.0. $\mathrm{P}<0.05$ was considered statistically significant.

\section{Results}

\section{The miRNA expression profile of renal exosomes in AKI model}

In order to explore the miRNA profile of renal exosomes in the acute kidney injury (AKI) model, AKI was induced by LPS administration as previously reported [19]. Levels of serum creatinine and urinary albumin-to-creatinine ratios were significantly increased in LPS-injected mice compared with control mice (Fig. 1a). Histologically, LPS induced significant renal tubular vacuolization, as well as CD68positive macrophage infiltration in the tubulointerstitium (Fig. 1b). Exosomes from the kidneys of AKI mice were then isolated for miRNA profile detection. TEM showed a typical size and shape of purified renal exosomes with membrane structures (Fig. 1c). Exosomes from control and LPS-treated mice had a mean particle size of $94 \pm 28.6 \mathrm{~nm}$ and $95 \pm 33.8 \mathrm{~nm}$, respectively (Fig. 1d). The exosomal protein markers Alix, CD9 and CD63 were readily detected by western blot analysis, as reported before [10].

We next characterized the miRNA signature of renal exosomes using the miRCURY LNA Array (version 11.0) system (Exiqon, Denmark). Renal exosomal miRNAs expression differed profoundly between LPS and PBStreated mice as demonstrated by the fold change and p-value as shown in Fig. 1e. Hierarchical clustering analysis of miRNA expression detected that of 1178 miRNAs, 176 (68 upregulated and 108 downregulated) were differentially expressed (Supplementary Fig. 1, Supplementary Table 1). Of these miRNAs, the top 10 miRNAs with remarkable upregulation and downregulation based on the fold change and $\mathrm{p}$ value in exosomes were shown in Fig. 1f. Among the top miRNAs, miR-19b-3p was previously reported to regulate inflammation [20], and it was selected for further studies.

To confirm the miRNA profile, we measured the expression of miR-19b-3p in renal exosomes via quantitative reverse transcription-polymerase chain reaction (qRT-PCR). A 2.73-fold increase of miR-19b-3p compared to controls was demonstrated ( $p<0.05$, Fig. 1g). To determine to what extent tubular exosomal miRNAs contribute to the expression in the whole kidney, exosomes were then isolated from tubular fraction of the kidney tissue. Interestingly, the upregulation of miR19b-3p was much more dramatic with 59.7 folds change compared to the whole kidney exosomes (Fig. 1h). Thus, miRNAs were selectively loaded into secreted exosomes in the kidneys, especially in TECs, in the AKI model.

\section{miR-19b-3p is increased in kidney exosomes in an adriamycin-induced proteinuric disease model}

To examine renal exosome secretion in a chronic kidney injury model, we subjected C57BL/6 J mice to adriamycin (ADR) injury. A single intravenous injection of ADR $(18 \mathrm{mg} / \mathrm{kg})$ was administered and mice were sacrificed at day 23 (Fig. 2a). As expected, mice injected with ADRdeveloped severe proteinuria 2 weeks after ADR injection (Fig. 2b). At 3 weeks after ADR injection, prominent tubular dilation accompanied by intraluminal protein casts and significant increases in mRNA expression of inflammatory cytokines, including MCP-1 and IL- 6 developed in ADR-injected mice (Fig. 2c, d). Moreover, the expression of p65 as well as the phosphorylated p65 (P-p65), one of the subunits of the nuclear factor- $\mathrm{kB}$ (NF$\kappa \mathrm{B})$ family, was upregulated in the diseased kidney (Fig. 2e). Of note, the number of kidney exosomes was also increased, as shown by western blot analysis with Alix, CD9 and CD63 (Fig. 2f). This was associated with a marked increase in the expression of miR-19b-3p in renal exosomes (Fig. 2g). Therefore, miR-19b-3p was enriched in kidney exosomes in a chronic kidney injury model. 

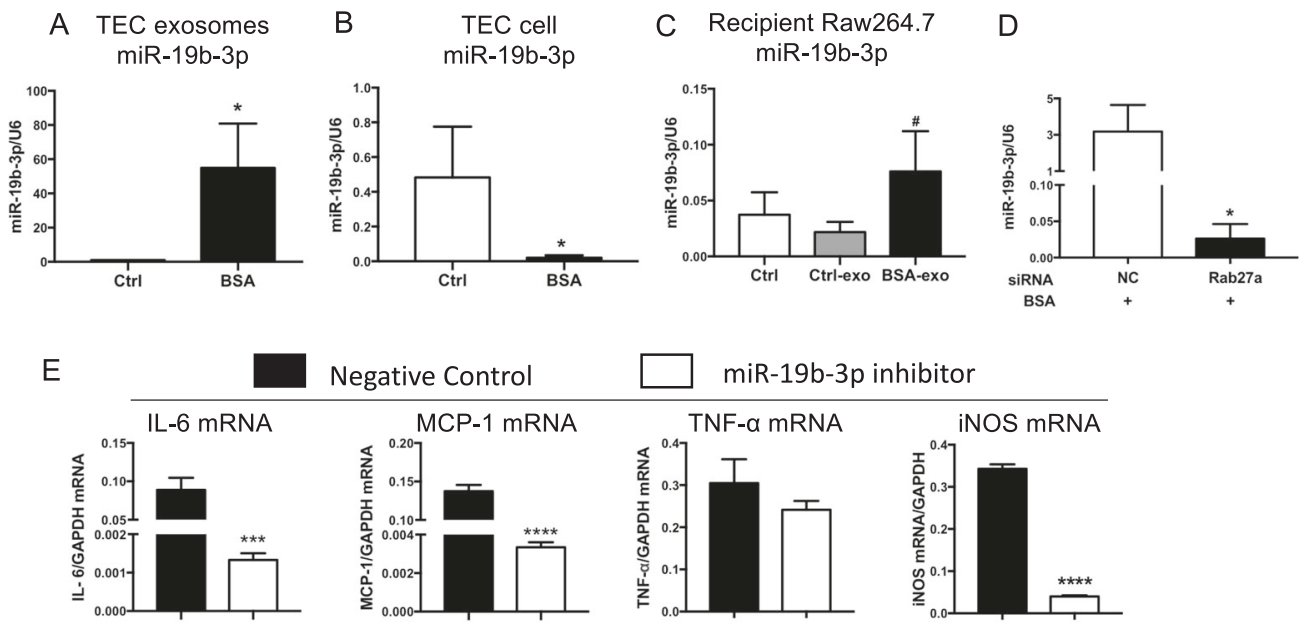

F Inhibitor NC miRNA-19b-3p
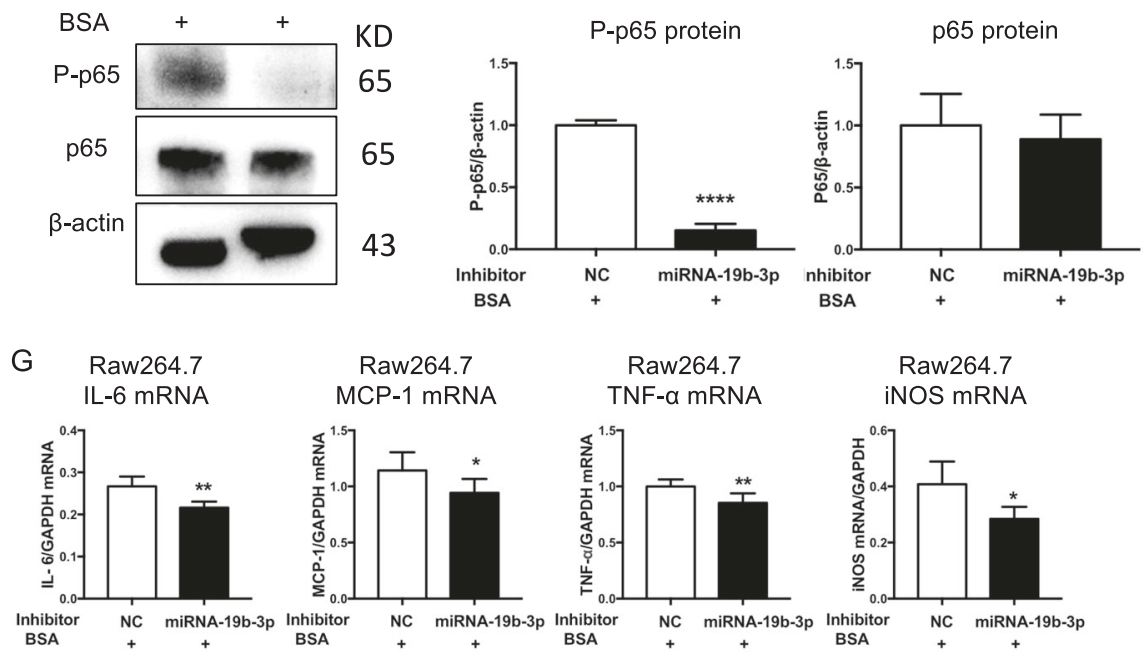

$\mathrm{H}$ Inhibitor NC miRNA-19b-3p
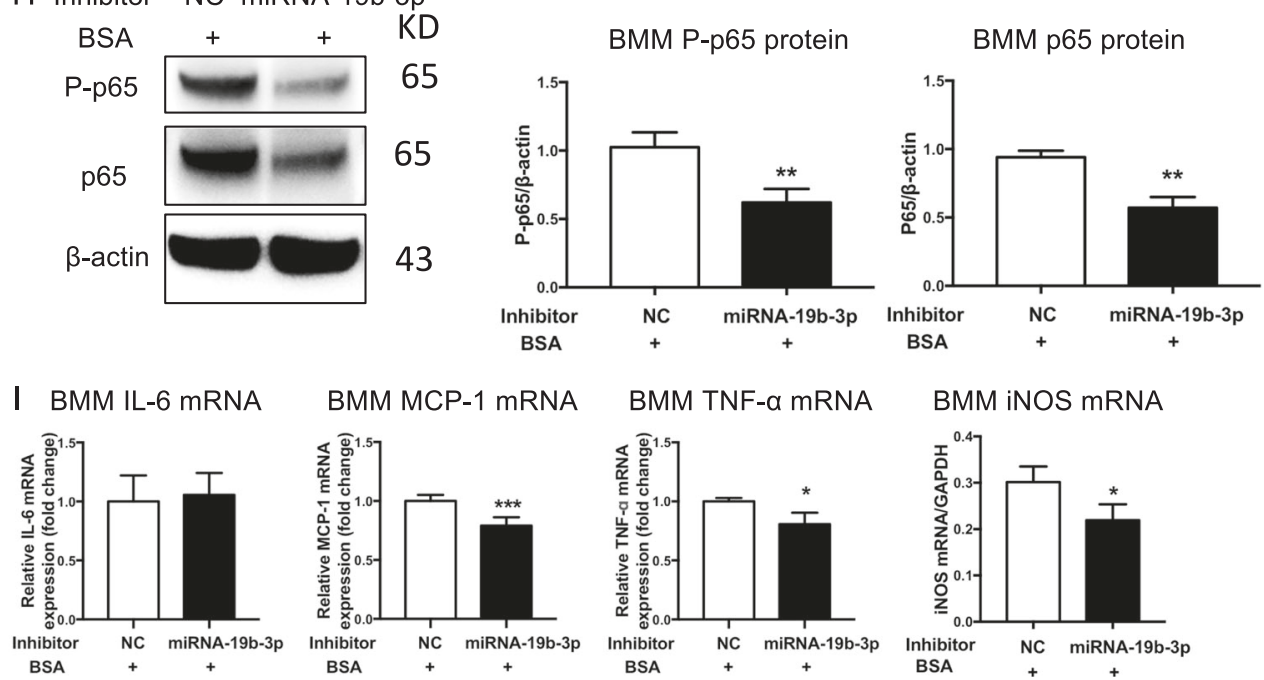
Fig. 4 TECs exosomes promoted M1 macrophage activation via miR19b-3p. a-c Expression of miR-19b-3p in TEC-derived exosomes $(* p=0.0225$ vs Ctrl TEC-derived exosomes), TEC cells $(* p=0.0491$ vs Ctrl TECs) and recipient Raw264.7 macrophages treated with TECderived exosomes $\stackrel{\#}{*} p=0.0495$ vs Ctrl Raw264.7 macrophages), respectively. d Expression of miR-19b-3p in TEC-derived exosomes with Rab27a silencing. ${ }^{*} p=0.0269$ vs NC. e Inflammatory cytokines and iNOS expression in macrophages treated with exosomes from TECs transfected with miR-19b-3p inhibitor or NC. Exosomes were purified and applied to recipient Raw264.7 macrophages. Upregulation of IL6, MCP-1 and iNOS mRNA was remarkably reversed in TECexo with miR-19b-3p inhibitor. $* * * p=0.0007$; $* * * * p<0.0001$ vs cells with NC inhibitor. f, g Raw264.7 macrophages were transfected with NC or miR-19b-3p inhibitor in the presence of BSA. f Representative western blotting of three independent experiments, and quantification of P-p65(****p 0.0001 versus NC) and p65 in Raw264.7 macrophages. g Inflammatory cytokine (IL6, $* * p=0.0025$; MCP- $1, * p=0.0463$; TNF- $\alpha, * * p=0.0072$ vs NC) and iNOS mRNA expression $(* * p=0.0299$ vs $\mathrm{NC}$ ) in Raw264.7 macrophages was detected by RT-PCR. h, i Bone marrow-derived macrophages (BMMs) were transfected with $\mathrm{NC}$ or miR-19b-3p inhibitor in the presence of BSA. h Representative western blotting of three independent experiments, and quantification of P-p65 $(* * p=0.0089$ versus BMMs with $\mathrm{NC}$ inhibitor), $\mathrm{p} 65{ }^{* *} p=0.0023$ versus BMMs with $\mathrm{NC}$ inhibitor) in BMMs after transfection. I Inflammatory cytokine (IL6; MCP-1, ${ }^{* * *} p=0.00045$; TNF- $\alpha, p=0.0277$ vs NC) and iNOS $(* p=0.014$ vs NC) mRNA expression in BMMs was detected by RTPCR. NC negative control, Ctrl exo exosomes from TECs without BSA, BSA-exo exosomes from TECs with BSA treatment. Data presented as mean \pm S.E.M. of three independent experiments. $p$ values were calculated using unpaired Student's $t$-test

\section{Increasing internalization of TEC-derived exosomes promotes M1 macrophage activation}

In order to understand the effect of TEC exosomes on macrophage activation, exosomes were then purified from the culture medium of TECs. TEM showed typical vesicle structure and size of exosomes as detected by phosphotungstic acid staining (Fig. 3a). The average size of the vesicles purified from TECs treated with BSA or untreated were $121.2 \pm 42.2 \mathrm{~nm}$ and $125.0 \pm 44.0 \mathrm{~nm}$, respectively (Fig. 3b). Western blot analysis showed that these exosomes were positive for Alix, CD9 and CD63, and BSA-treated TECs secreted more exosomes than control group (Fig. 3c).

To explore the communication between TECs and macrophages, we established a co-culture system where DiO-labeled TECs were cultured together with macrophages, separated by a $0.4 \mu \mathrm{m}$ Transwell filter, which would avoid the direct cell-cell contact or the shift of larger particles. The internalization of fluorescent exosomes was observed inside the naïve macrophages and was enhanced by BSA treatment (Fig. 3d). In addition, to further confirm the direct transfer of exosomes between cells, we isolated and purified DiO-labeled TECderived exosomes by ultracentrifugation and applied them to macrophages. As expected, macrophages internalized more fluorescently labeled exosomes when purified from BSA-treated TECs (Fig. 3e). Interestingly, as the result of direct communication through exosomes, levels of p65 and P-p65 protein were increased in exosome-exposed macrophages. This was associated with a significant upregulation of inflammatory cytokines including MCP-1, IL-1 $\beta$, IL-6 and TNF- $\alpha$ (Fig. 3f, g). Notably, both mRNA and protein expression of iNOS, a marker of M1 macrophages, was sharply increased in BSA-treated TEC exosomes, indicating that the TEC exosomes polarize macrophage toward a proinflammatory M1 phenotype.

\section{TECs exosomes promote M1 macrophage activation via miR-19b-3p}

In cultured TECs exposed to BSA, miR-19b-3p was enriched in secreted exosomes but decreased in cells, which indicated the relocation of miR-19b-3p from cellular compartment to exosomes (Fig. 4a, b). Interestingly, macrophages treated with TEC-derived exosomes exhibited high levels of miR-19b-3p (Fig. 4c). These results indicated that BSA could enhance miR-19b-3p loading into exosomes and transfer to recipient macrophages. Previous studies suggested that Rab27a is essential for cargo secretion via exosomes. To identify the effect of the Rab27a-dependent pathway on the secretion of miR-19b-3p, Rab27a siRNA was transfected in TECs, followed by BSA treatment. Notably, TEC-derived exosomal miR-19b-3p sharply decreased after transfected with Rab27a siRNA (Fig. 4d). To investigate whether miR-19b-3p mediates the effects of TEC exosomes in M1 macrophage activation, exosomes from TECs with miR-19b-3p inhibition using anti-miR oligonucleotides were isolated and applied to macrophages. Notably, inhibition of miR-19b-3p significantly decreased the expression levels of IL-6, MCP-1 and iNOS mRNA in recipient macrophages treated with TEC exosomes (Fig. 4e).

Moreover, treatment of BSA-exposed Raw264.7 macrophages with an miR-19b-3p inhibitor ameliorated $\mathrm{NF}-\kappa \mathrm{B}$ activation and reversed the upregulation of inflammatory cytokines, including IL-6, MCP-1, TNF$\alpha$ and iNOS (Fig. 4f, g). The same findings were alsoconfirmed in isolated bone marrow derived-macrophages (BMMs). (Fig. 4h, i), demonstrating that TEC-exosomes promote M1 macrophage activation via an miR-19b-3p$\mathrm{NF}-\kappa \mathrm{B}$ signaling-dependent pathway.

\section{Exosomal miR-19b-3p activates NF- $K B$ in macrophage via targeting SOCS-1}

Next, we sought to understand the mechanism through which miR-19b-3p promotes M1 macrophage activation. 
A

Position 292-299 of Socs1 3' UTR $5^{`}$ - . . CAUAUUCCCAGUAUCUUUGCACA . . . - $3^{\text {‘ }}$

mmu-miR-19b-3p 3‘- AGUCAAAACGUACCUAAACGUGU - 5،

Predicted by TargetScan Release 7.0

B Recipient Raw264.7

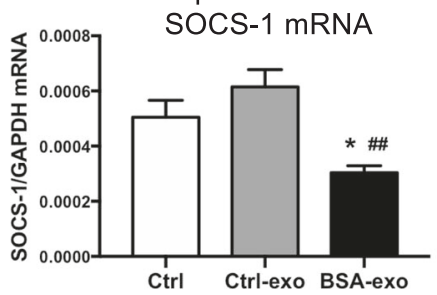

C

Mimic NC miR-19b-3p KD

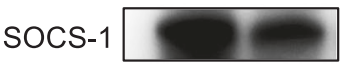

$\beta$-actin

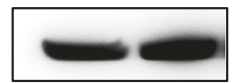

43

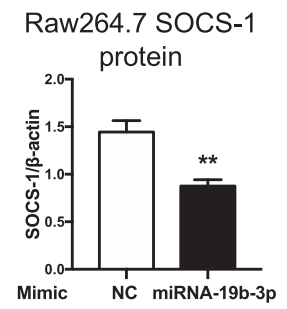

E

Inhibitor NC miR-19b-3p NC miR-19b-3p
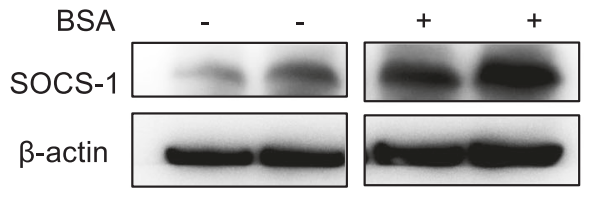

KD

F

Inhibitor NC miR-19b-3p NC miR-19b-3p
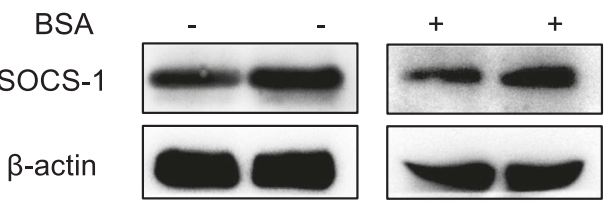

G

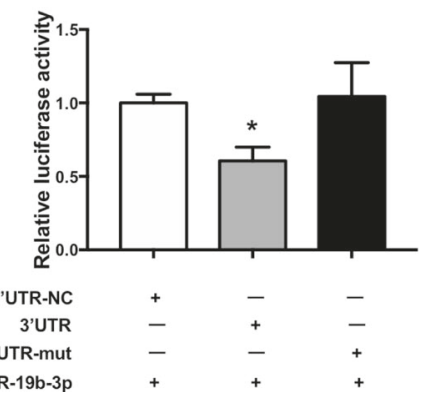

Recipient Raw264.7 SOCS-1 protein

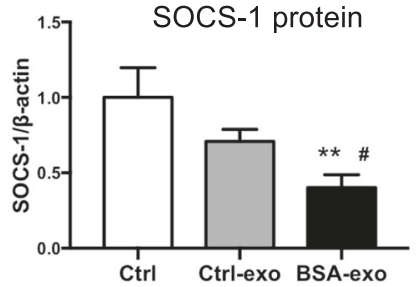

D

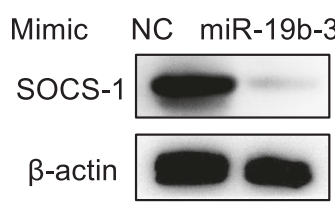

BMM SOCS-1 protein
36

43

Raw264.7 SOCS-1 protein

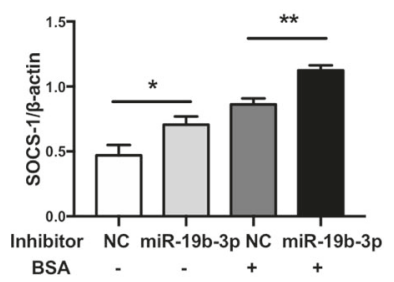

BMM SOCS-1 protein

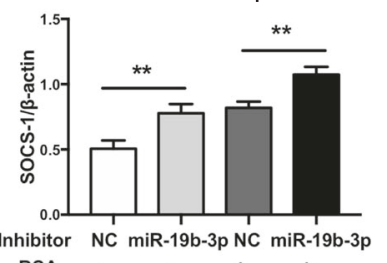

H $\square$ Negative control inhibitor $\square$ miR-19b-3p inhibitor

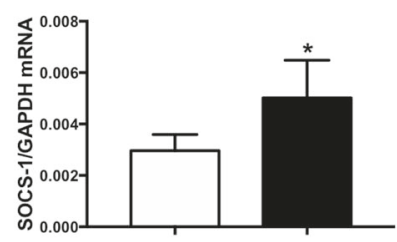


Fig. 5 Exosomal miR-19b-3p activates NF- $\kappa$ B in macrophage via targeting SOCS-1. a Shown are miR-19b-3p binding sites in the SOCS-1 3' UTR. The binding sequence of miR-19b-3p are located at nucleotide 292 to 299 from the 3' UTR as predicted in TargetScan Release 7.0. b mRNA and protein expression of SOCS-1 in recipient Raw264.7 macrophages treated with TEC-exo by RT-PCR and western blot analysis. Representative blots and quantification showed significant repression of SOCS1 in BSA-exo group. ${ }^{*} p=$ $0.0344 ; * * p=0.0026$ vs Ctrl; ${ }^{\#} p=0.0261 ;{ }^{\# \#} p=0.0054$ vs Ctrlexo. c, d Overexpression of miR-19b-3p in Raw264.7 macrophages and BMMs induced significant reduction of SOCS-1 protein revealed by western blot. $* * p=0.0035$; $* * * *<<0.0001$ vs macrophages with NC mimic. e, f Inhibition of miR-19b-3p in Raw264.7 macrophages and BMMs induced significant upregulation of SOCS-1 protein. ${ }^{*} p=0.0129 ; * * p=0.0058$ vs Raw 264.7 macrophages with NC inhibitor, $* * p=0.0049$ and 0.0019 vs BMMs with $\mathrm{NC}$ inhibitor. $N C$ negative control. BSA. g Luciferase reporter assay was performed with constructs with negative control (NC), SOCS-1 $3^{\prime}$ untranslated region(UTR) or SOCS-1 $3^{\prime}$-UTRmutant. 293T cells were transfected with these constructs and the miR-19b-3p overexpressed plasmid. $* p=0.032$, compared with the NC+miR-19b-3p group. h The expression of SOCS-1 in macrophages treated with exosomes from TECs transfected with miR-19b-3p inhibitor or NC. $* p=0.0416$, compared with cells with NC inhibitor. Data presented as mean \pm S.E.M. of three independent experiments. $p$ values were calculated using unpaired Student's t-test for two groups comparison. For multiple comparison, p-values were calculated using one-way ANOVA test, Bornferroni-corrected $\alpha$ threshold was used for statistical significance

Based on bioinformatics analysis, SOCS-1 (suppressor of cytokine signaling) was found to be the putative target genes for miR-19b-3p and was also a negative regulator of NF- $\mathrm{KB}$ signaling pathway (Fig. 5a).To test whether exosomal miR-19b-3p targets SOCS-1 in recipient macrophages, miR-19b-3p-enriched exosomes from BSAtreated TECs were applied to macrophages. The results showed that both mRNA and protein expressions of SOCS-1 were concomitantly inhibited (Fig. 5b) in recipient macrophages. In both Raw264.7 macrophages and BMMs, overexpression of miR-19b-3p suppressed the expression of SOCS-1, while miR-19b-3p inhibitor significantly upregulated expression of SOCS-1 (Fig. 5c-f). Next, a dual luciferase reporter assay was conducted, and the results showed that the activity of luciferase reporters was markedly reduced by miR-19b$3 p$ overexpression, while the activity of the SOCS-1 3'UTR - mutant luciferase reporter was not affected, demonstrating that SOCS-1 was the direct target gene of miR-19b-3p (Fig. 5g). Furthermore, the reduction of SOCS-1 was detected in RAW264.7 exposed to exosomes from tubular epithelial cells transfected with miR19b-3p inhibitor (Fig. 5h). Thus, TEC exosomal miR$19 b-3 p$ may activate NF- $\kappa B$ signaling in macrophage via targeting SOCS-1.

\section{TEC exosomes promote tubulointerstitial inflammation through miR-19b-3p in vivo}

We next investigated the functional role of miR-19b-3p in TEC exosomes in vivo. TECs treated with or without NC or miR-19b-3p inhibitor were stimulated with BSA and exosomes were isolated and transferred into mice via renal injection. Mice were euthanized $24 \mathrm{~h}$ after injection. Exosomes from BSA treated TECs caused significant tubular injury and tubulointerstitial inflammation, while exosomes from miR-19b-3p-depleted TECs resulted in less severe tubular injury and decreased $\mathrm{F} 4 / 80$ positive macrophage infiltration (Fig. 6a). Accordingly, BSAtreated TEC-derived exosomes increased the expression of renal inflammatory cytokine compared to control mice, while mice injected with TEC-derived exosomes with miR-19b-3p inhibition showed decreased levels of renal inflammation by downregulating IL- 6 and MCP-1 mRNA expression (Fig. 6b). The results suggested that miR-19b-3p can be functionally delivered to the renal tissue and trigger renal inflammation via TEC-derived exosomes.

\section{Increased exosomal miR-19b-3p in urine from patients with diabetic nephropathy correlates with the severity of tubulointerstitial inflammation}

To confirm our findings clinically, we isolated and purified exosomes from the urine samples of patients with type II diabetes (T2DM, $n=15$ ) and biopsy-proven diabetic nephropathy (DN) $(n=28)$. The clinical characteristics of the cohorts are listed in Table 2. The median levels of BUN and $\mathrm{SCr}$ were significantly elevated and eGFR was decreased in DN patients compared with T2DM patients. The median level of urinary ACR was significantly increased in patients with DN, whereas T2DM patients were negative for urinary ACR. Compared with T2DM patients, the protein levels of exosomal markers were increased in urinary exosomes from DN patients. Importantly, AQP2, the tubular epithelial marker, was also detected in exosomes purified from urinary samples of DN patients, indicating the TEC source of the urinary exosomes (Fig. 7a). Notably, RTPCR analysis revealed a marked elevation of miR-19b-3p expression in the urinary exosomes from DN patients compared with T2DM patients (Fig. 7b). Moreover, the expression of urinary exosomal miR-19b-3p was positively correlated with the severity of albuminuria (Fig. 7c), which was associated with a marked tubulointerstitial inflammation (Fig. 7d). Interestingly, DN patients with lower levels of urinary exosomal miR-19b-3p had higher levels of SOCS-1, as shown by immunofluorescence staining in the kidney (Fig. 7e). 
A

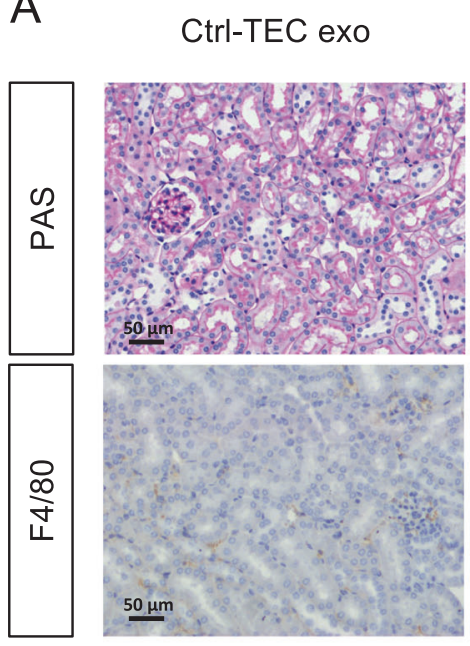

BSA-TEC exo with NC inhibitor
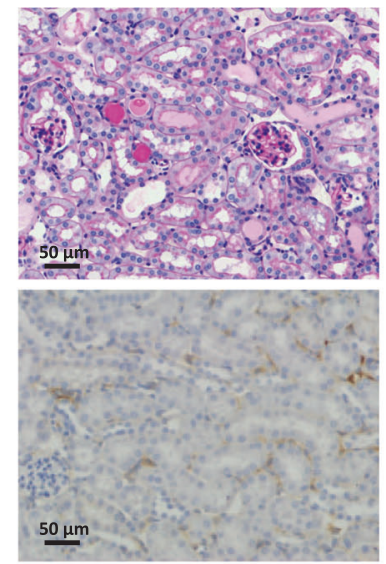

BSA- TEC exo with

miRNA-19b-3p inhibitor
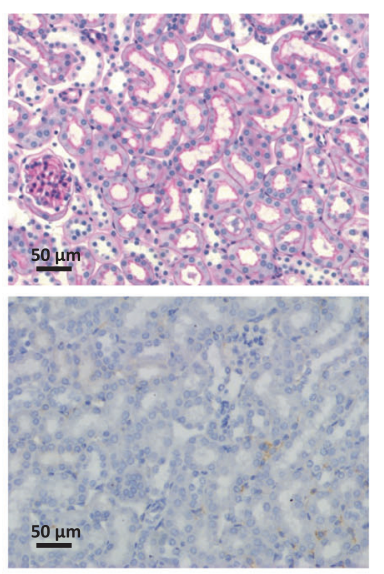

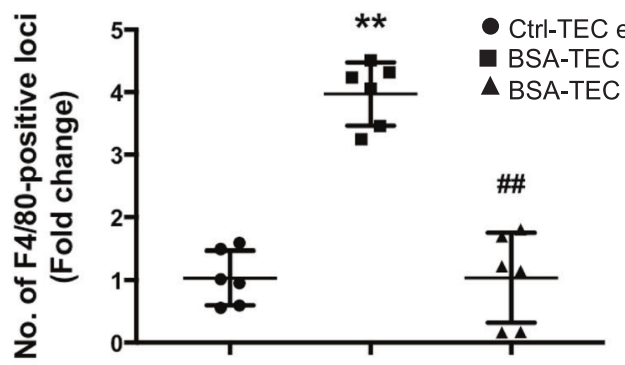

B

- Ctrl-TEC exosomes

- BSA-TEC exosomes with NC inhibitor

A BSA-TEC exosomes with miR-19b-3p inhibitor

IL-6 mRNA

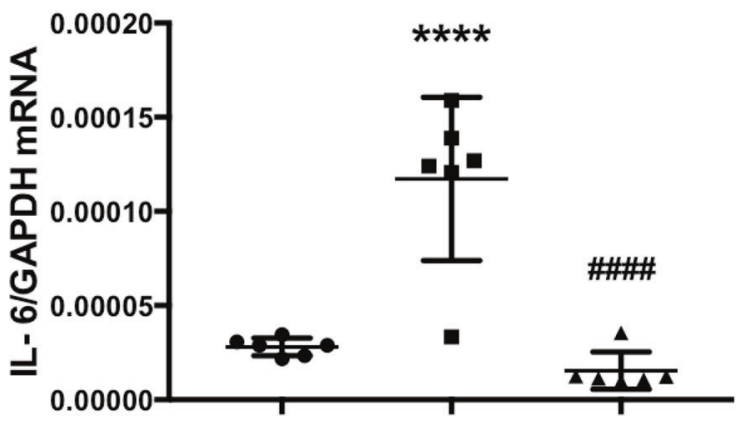

Fig. 6 TEC exosomes promoted tubulointerstitial inflammation through miR-19b-3p in vivo. TECs were cultured and treated with BSA or PBS for $24 \mathrm{~h}$ after transfection of NC or miR-19b-3p inhibitor. Exosome was transferred to $\mathrm{BALB} / \mathrm{c}$ mice through renal injection. Mice were euthanized $24 \mathrm{~h}$ after injection ( $n=6$ for each group). a Histologic (PAS staining) changes and F4/80 positive macrophage infiltration in the kidney. The number of F4/80 positive loci were quantified. $* * p=0.0036$ vs mice with Ctrl-exo and ${ }^{\# \#} p=0.0036$ vs mice with NC inhibitor. b RT-PCR analysis showed mRNA

\section{Discussion}

Tubulointerstitial inflammation is a common pathological feature of acute and chronic kidney injury and determines

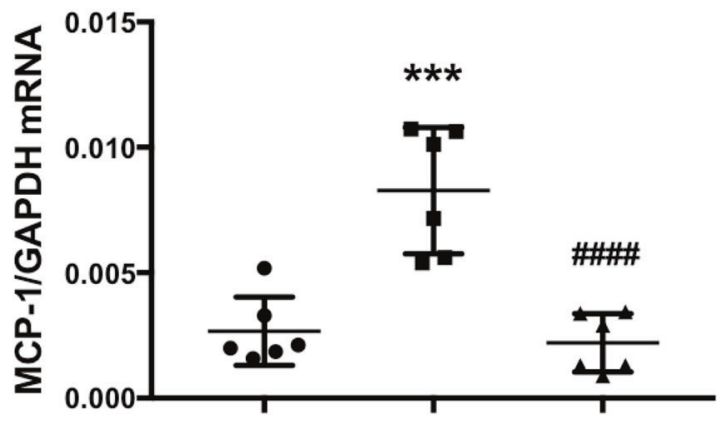

expression of renal inflammatory cytokines (IL-6, MCP-1) in the kidney. BSA-exo NC group showed significant upregulation of IL-6 $(* * * * p<0.0001$ vs mice with Ctrl-exo, \#\#\# $p<0.0001$ vs with NC inhibitor) MCP-1(***p $=0.0002$ vs mice with Ctrl-exo, ${ }^{\# \# \# ~} p<0.0001$ vs with NC inhibitor) mRNA, while miR-19b-3p inhibitor reverse the upregulation significantly. Scale bar, $50 \mu \mathrm{m}$. For multiple comparison, $p$-values were calculated using one-way ANOVA test, Bornferronicorrected $\alpha$ threshold was used for statistical significance. Ctrl control, $N C$ negative control

the outcome of kidney disease. However, the mechanism by which the initial injury of TECs drives interstitial inflammation remains unclear. In this study, we demonstrate a novel mechanism through which the exosomal miR-19b-3p/ 
Table 2 Human subject characteristics of T2DM and DN patients

\begin{tabular}{lcc}
\hline & T2DM $(n=15)$ & $\mathrm{DN}(n=28)$ \\
\hline Males & $8(53 \%)$ & $15(53 \%)$ \\
Age(years) & $52(42-68)$ & $60(54-69)$ \\
BMI(kg/m²) & $25.79(23.61-28.46)$ & $24.65(22.24-27.93)$ \\
Total cholesterol(g/L) & $4.76(4.28-5.55)$ & $4.44(3.74-6.79)$ \\
Triglyceride(mg/dL) & $2.47(1.55-3.87)$ & $1.8(1.37-2.22)$ \\
BUN(mmol/L) & $5.85(4.93-9)$ & $11.6(6.4-16.5)^{* *}$ \\
SCr(umol/L) & $85(70.5-107)$ & $184(98-249)^{*}$ \\
UA(umol/L) & $328(284-366)$ & $367(328-475)$ \\
Plasma albumin(g/L) & $42(40-43)$ & $31(27-35)^{* * * *}$ \\
eGFR(ml/min/1.73 m2) & $83.72(58.29-99.62)$ & $32.47(19.40-73.18)^{*}$ \\
Urinary ACR (mg/g) & 0 & $579(208-707)^{* *}$ \\
\hline BMI body
\end{tabular}

$B M I$ body mass index, $B U N$ blood urea nitrogen, $S C r$ serum creatinine, $U A$ uric acid, eGFR estimated glomerular filtration rate. eGFR has been calculated using the CKD-EPI Creatinine formula (ml/ s per $1.73 \mathrm{~m} 2$ ). Values are expressed as median (IQR) (Mann-Whitney $U$ test; $* p<0.05 ; * * p<0.01 ; * * * * p<0.0001)$

SOCS1 axis mediated cellular communication between TECs and macrophages and subsequently induced tubulointerstitial inflammation (Fig. 8). Our findings may provide a novel molecular target for the treatment of tubulointerstitial inflammation.

Firstly, microarray analysis revealed that miRNAs, especially miR-19b-3p, were selectively loaded into exosomes in TECs in both LPS-induced acute kidney injury and ADR-induced chronic kidney injury models. Exosomes purified from kidney tubules in vivo and cultured TECs had a remarkably high expression of miR-19b-3p compared to that of controls. Previous studies showed that miRNA expression in TECs changed under pro-inflammatory conditions and with fibrosis, which limited or promoted the injury response [17, 21-23]. Specific miRNAs might be actively sorted into exosomes under different pathological conditions to convert the regulatory roles of exosomes [24]. However, the excretion of miRNAs in TEC exosomes has not been investigated in kidney disease. This study clearly showed the significant differential profile of exosomal miRNAs in TECs and indicated the potential role of exosomal miRNA in kidney disease.

Next, the functional impact of miRNA in TEC exosomes in the setting of interstitial inflammation was investigated in this study. We demonstrated that TEC exosomes were capable of triggering M1 macrophage activation via the miR-19b-3p-dependent mechanism, which provided a new insight into the mechanism of macrophage polarization in kidney disease.
It is well-known that phenotype switching of macrophages can be triggered by cytokine or ligand-receptor interaction during kidney injury [25-27]. Our studies revealed that exosome-mediated cellular communication is an alternative approach to initiate M1 macrophage activation. Since the first report that exosomes contain both mRNA and microRNA, which can be delivered to another cell as functional cargoes [12], exosomal RNAs have been regarded as new paradigm in gene regulation. Exosome-associated miRNAs can regulate target gene expression in recipient cells upon internalization. Alternatively, miRNAs released by cell EVs have been shown to bind to TLRs and contribute to inflammation [28]. Our results here suggest that exosomal miR-19b-3p excreted from TECs could exert its regulatory role through vesicle mediated internalization by macrophages.

More importantly, as shown in this study, adoptive transfer of exosomes enriched with miR-19b-3p induced renal inflammation. In contrast, exosomes derived from TECs with inhibition of miR-19b-3p abrogated renal inflammation. Thus, miR-19b-3p could be transferred to macrophages as a functional molecule from TEC exosomes and promote tubulointerstitial inflammation. miR-19b-3p is known to play key roles in oncogenic processes and is often overexpressed in several cancers, including pancreatic cancer and breast cancer [29, 30]. The validated targets of miR-19b-3p are related to cell death, the immune response, and proliferation [31, 32]. Our study here reported a novel function of miR-19b-3p in exosomes as a regulator of macrophage activation and tubulointerstitial inflammation in kidney disease. The exosome-mediated inflammatory pathway may be a new mechanism responsible for the development of renal inflammation by promoting TECmacrophage communication.

The mechanism through which exosomal miR-19b-3p regulated the M1 macrophage activation was further investigated by demonstrating its repressive effect on the expression of SOCS-1, a negative regulator of NF- $\mathrm{NB}$ signaling pathways. SOCS-1 was initially identified as an intracellular negative-feedback molecule that ubiquitinated RelA/p65 [33, 34]. The data here showed a significant reduction of SOCS-1 protein in macrophages when treated with TECs exosomes enriched with miR-19b-3p. miR-19b-3p inhibitors or mimics could remarkably alter SOCS-1 protein expression. Thus, we suggested that the exosome/miR-19b-3p/SOCS1 axis plays a critical pathogenic role in M1 macrophage activation and tubulointerstitial inflammation.

Interestingly, we demonstrated the remarkable increase of exosome excretion in DN patients compared to patients with T2DM without albuminuria. Importantly, urinary exosomes were demonstrated to originated 


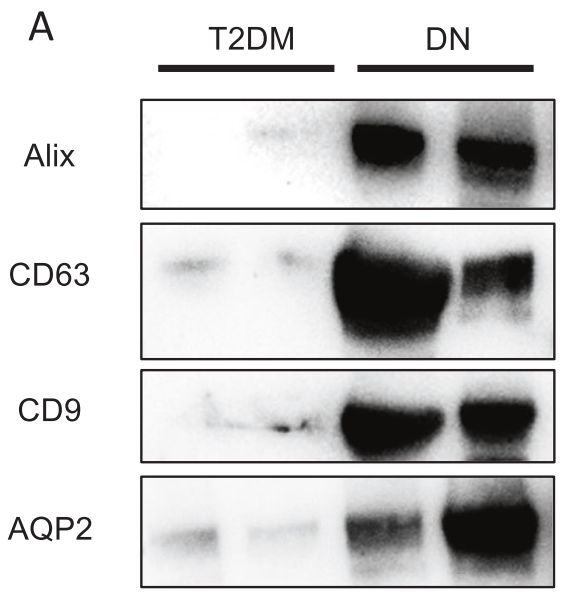

B

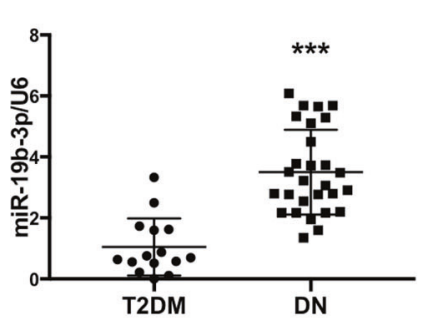

C

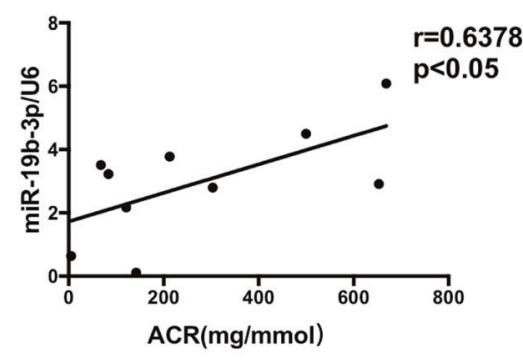

The level of urinary exosomal miR-19b-3p

$E$

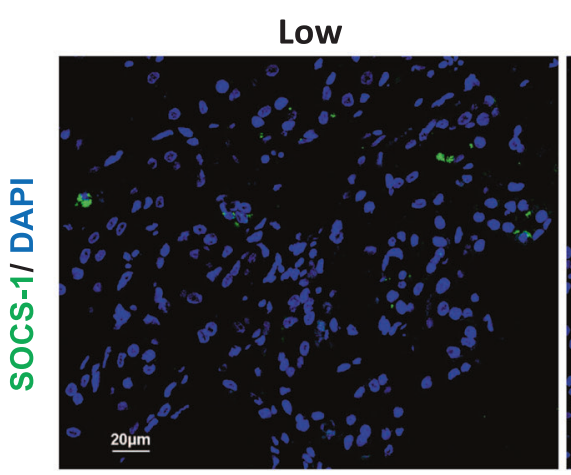

90

25

42

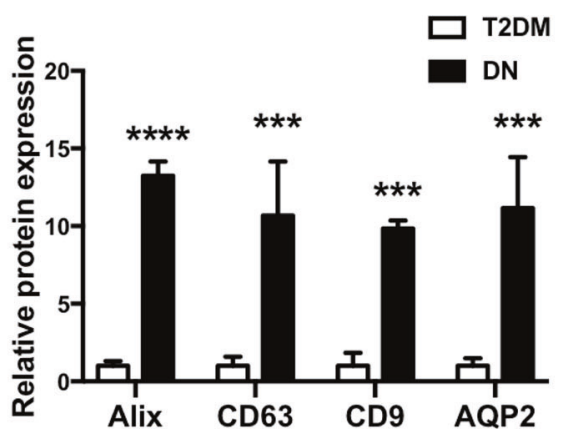

D Tubulointerstitial inflammation

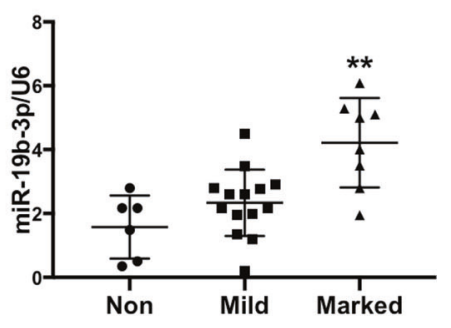

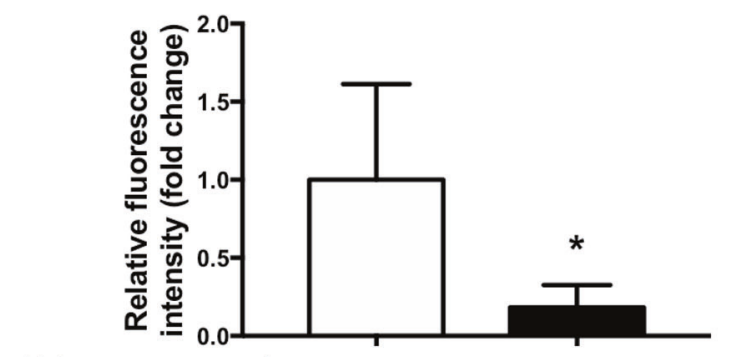

Urinary exosomal Low level High level miR-19b-3p

partly from TECs, as AQP2 protein was readily detected in the exosome fraction. We found that the expression of urinary exosomal miR-19b-3p was positively correlated with the severity of albuminuria. Moreover, the level of miR-19b-3p in urinary exosomes correlated with the severity of tubulointerstitial 
Fig. 7 Increased exosomal miR-19b-3p in urine from patients with diabetic nephropathy (DN) were associated with the severity of tubulointersitial inflammation. a Representative western blotting and quantification of exosomal markers (Alix, CD9 and CD63) and tubular marker AQP2 in urinary exosomes from T2DM and DN patients. Alix, $* * * * p<0.0001$; CD63, $* * * p=0.0003$; CD9, $* * * p=0.0009$; AQP2, $* * * p=0.0002$ vs T2DM patients. b Urinary exosome miR-19b-3p in DN patients. The expression of miR-19b-3p in urinary exosomes of DN patients $(n=28)$ was significantly higher than type 2 diabetes (T2DM) patients $(n=15) . * * * \mathrm{p}=0.001$ compared with T2DM patients. c Plots of correlation between miR-19b-3p in urinary exosomes and urinary ACR in DN patients $(r=0.6378, p<0.05)$. $\mathbf{d}$ The level of miR-19b-3p in urinary exosomes of DN patients with normal $(0, n=6)$, mild $(<25 \%, n=14)$ or marked $(>25 \%, n=8)$ interstitial inflammation. Patients with marked interstitial inflammation showed the highest levels of urinary exosomal miR-19b-3p (**p $=0.0089$ vs DN patients without tubulointerstitial inflammation). e Immunofluorescence of renal biopsies from patients with $\mathrm{DN}$ by using antiSOCS-1(green). DN patients with lower level of miR-19b-3p in urinary exosomes shows higher expression of SOCS-1 in the tubulointerstitium(left). Relative fluorescence intensity of SOCS-1 was shown in lower panel $(* p=0.012)$. $p$ values were calculated using unpaired Student's $t$-test for two groups comparison. For multiple comparison, $p$-values were calculated using one-way ANOVA test, Bornferroni-corrected $\alpha$ threshold was used for statistical significance. Scale bar, $20 \mu \mathrm{m}$

inflammation. Patients with higher levels of urinary exosomal miR-19b-3p showed a lower expression of SOCS-1 in the kidney. Thus, these clinical data further support our findings that TEC exosomal miR-19b-3p contributed to tubulointerstitial inflammation by targeting SOCS-1 in macrophages and might serve as potential biomarker of kidney disease.

In conclusion, we have demonstrated that exosomal miR19b-3p mediated the cross-talk between TECs and macrophages and contributed to M1 macrophage activation. The exosome/miR-19b-3p/SOCS1 axis played a critical role in the development of tubulointerstitial inflammation. The findings suggested that exosomal miR-19b-3p might represent a new therapeutic target for the progression of kidney disease.

Acknowledgements This study is supported by grants from the National Natural Scientific Foundation of China (grant number 81720108007, 81470922, 31671194,), National Key R\&D Program of China (2018YFC1314002), the Fundamental Research Funds for the Central Universities, and Research Grants Council of Hong Kong (GRF 14121816).

\section{Compliance with ethical standards}

Conflict of interest The authors declare that they have no conflict of interest.

Publisher's note: Springer Nature remains neutral with regard to jurisdictional claims in published maps and institutional affiliations.

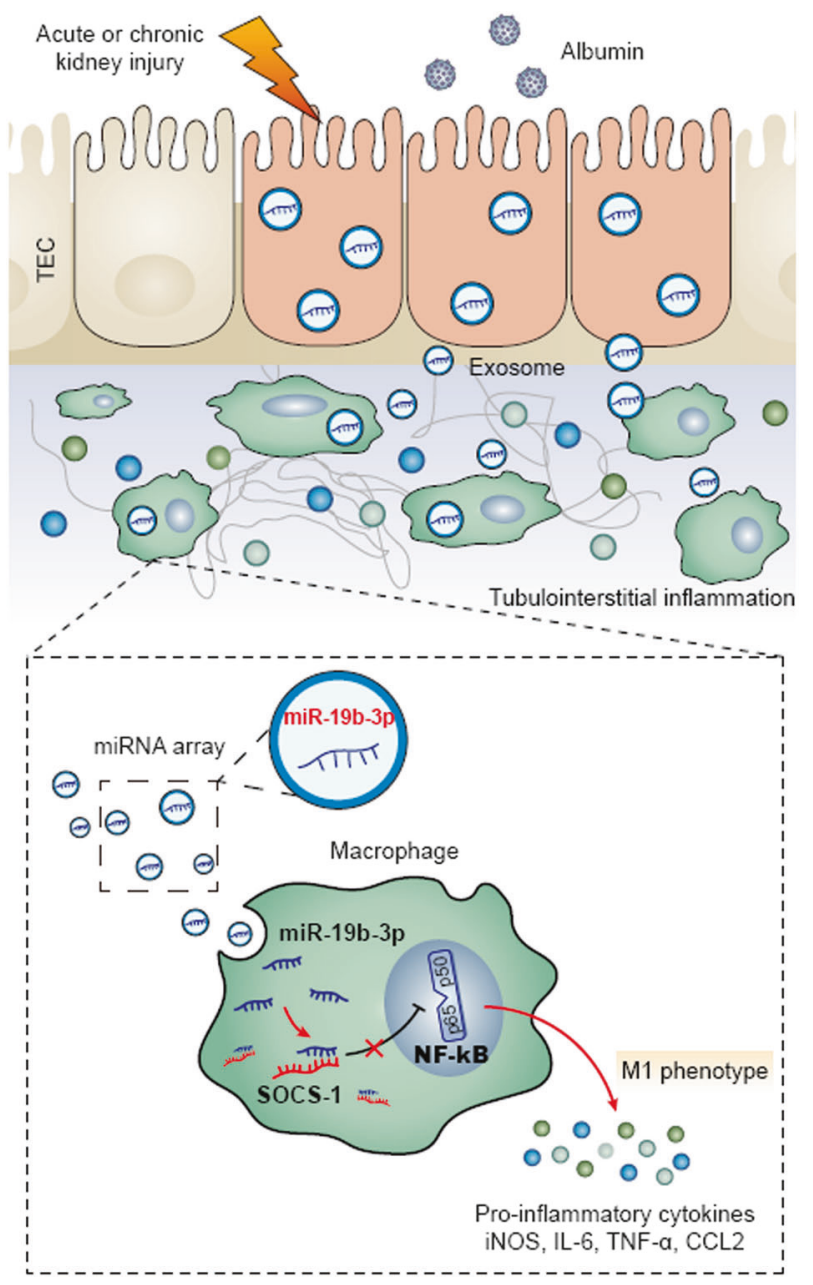

Fig. 8 Working model. Tubular epithelial cells (TECs) secrete exosomes containing miR-19b-3p upon injury, which are internalized by macrophages. And miR-19b-3p enhance NF-Kb activity through directly targeting SOCS-1, promoting the M1 macrophage activation. The communication between TECs and macrophages via exosomalmiR-19b-3p augmented the initial injury signal from TECs and promoted the development of tubulointerstitial inflammation

\section{References}

1. Liu BC, Tang TT, Lv LL, Lan HY. Renal tubule injury: a driving force toward chronic kidney disease. Kidney Int. 2018;93: 568-79.

2. Bonventre JV. Dedifferentiation and proliferation of surviving epithelial cells in acute renal failure. J Am Soc Nephrol. 2003;14:55S-61.

3. Smith SF, Hosgood SA, Nicholson ML. Ischemia-reperfusion injury in renal transplantation: 3 key signaling pathways in tubular epithelial cells. Kidney Int. 2019;95:50-56.

4. Tan RJ, Zhou D, Liu Y. Signaling crosstalk between tubular epithelial cells and interstitial fibroblasts after kidney injury. Kidney Dis. 2016;2:136-44.

5. Wang Y, Harris DC. Macrophages in renal disease. J Am Soc Nephrol. 2011;22:21-27.

6. Thebaud B, Stewart DJ. Exosomes: cell garbage can, therapeutic carrier, or trojan horse? Circulation. 2012;126:2553-5. 
7. Harding C, Heuser J, Stahl P. Receptor-mediated endocytosis of transferrin and recycling of the transferrin receptor in rat reticulocytes. J cell Biol. 1983;97:329-39.

8. H Rashed M, Bayraktar E, K Helal G, Abd-Ellah MF, Amero P, Chavez-Reyes A, et al. Exosomes: from garbage bins to promising therapeutic targets. Int J Mol Sci. 2017;18: pii: E538. https://doi. org/10.3390/ijms 18030538 .

9. Tkach M, Thery C. Communication by extracellular vesicles: where we are and where we need to go. Cell. 2016;164:1226-32.

10. Lv LL, Feng Y, Wen Y, Wu WJ, Ni HF, Li ZL, et al. Exosomal CCL2 from tubular epithelial cells is critical for albumin-induced tubulointerstitial inflammation. J Am Soc Nephrol. 2018;29: 919-35.

11. Squadrito ML, Baer C, Burdet F, Maderna C, Gilfillan GD, Lyle R, et al. Endogenous RNAs modulate microRNA sorting to exosomes and transfer to acceptor cells. Cell Rep. 2014;8:1432-46.

12. Valadi H, Ekstrom K, Bossios A, Sjostrand M, Lee JJ, Lotvall JO. Exosome-mediated transfer of mRNAs and microRNAs is a novel mechanism of genetic exchange between cells. Nat Cell Biol. 2007;9:654-9.

13. Vinas JL, Burger D, Zimpelmann J, Haneef R, Knoll W, Campbell $\mathrm{P}$, et al. Transfer of microRNA-486-5p from human endothelial colony forming cell-derived exosomes reduces ischemic kidney injury. Kidney Int. 2016;90:1238-50.

14. Alexander M, Hu R, Runtsch MC, Kagele DA, Mosbruger TL, Tolmachova $\mathrm{T}$, et al. Exosome-delivered microRNAs modulate the inflammatory response to endotoxin. Nat Commun. 2015;6: 7321-36.

15. Lorenzen JM, Kaucsar T, Schauerte C, Schmitt R, Rong S, Hubner A, et al. MicroRNA-24 antagonism prevents renal ischemia reperfusion injury. J Am Soc Nephrol. 2014;25:2717-29.

16. Trionfini P, Benigni A. MicroRNAs as master regulators of glomerular function in health and disease. J Am Soc Nephrol. 2017;28:1686-96.

17. Liu XJ, Hong Q, Wang Z, Yu YY, Zou X, Xu LH. MicroRNA34a suppresses autophagy in tubular epithelial cells in acute kidney injury. Am J Nephrol. 2015;42:168-75.

18. Li J, Zelenin S, Aperia A, Aizman O. Low doses of ouabain protect from serum deprivation-triggered apoptosis and stimulate kidney cell proliferation via activation of NF-kappaB. J Am Soc Nephrol. 2006;17:1848-57.

19. Reiser J, von Gersdorff G, Loos M, Oh J, Asanuma K, Giardino L, et al. Induction of B7-1 in podocytes is associated with nephrotic syndrome. J Clin Investig. 2004;113:1390-7.

20. Gantier MP, Stunden HJ, McCoy CE, Behlke MA, Wang D, Kaparakis-Liaskos M, et al. A miR-19 regulon that controls NFkappaB signaling. Nucleic Acids Res. 2012;40:8048-58.
21. Jiang L, Qiu W, Zhou Y, Wen P, Fang L, Cao H, et al. A microRNA-30e/mitochondrial uncoupling protein 2 axis mediates TGF-beta1-induced tubular epithelial cell extracellular matrix production and kidney fibrosis. Kidney Int. 2013;84:285-96.

22. Pan J, Zhang J, Zhang X, Zhou X, Lu S, Huang X, et al. Role of microRNA-29b in angiotensin II-induced epithelial-mesenchymal transition in renal tubular epithelial cells. Int $\mathrm{J}$ Mol Med. 2014;34:1381-7.

23. Amrouche L, Desbuissons G, Rabant M, Sauvaget V, Nguyen C, Benon A, et al. MicroRNA-146a in human and experimental ischemic AKI: CXCL8-dependent mechanism of action. J Am Soc Nephrol. 2017;28:479-93.

24. He C, Zheng S, Luo Y, Wang B. Exosome theranostics: biology and translational medicine. Theranostics. 2018;8:237-55.

25. Rosin DL, Okusa MD. Dangers within: DAMP responses to damage and cell death in kidney disease. J Am Soc Nephrol. 2011;22:416-25.

26. Cao Q, Wang Y, Harris DC. Macrophage heterogeneity, phenotypes, and roles in renal fibrosis. Kidney Int Suppl. 2011;4:16-19.

27. Lv LL, Tang PM, Li CJ, You YK, Li J, Huang XR, et al. The pattern recognition receptor, Mincle, is essential for maintaining the M1 macrophage phenotype in acute renal inflammation. Kidney Int. 2017;91:587-602.

28. Buzas EI, Gyorgy B, Nagy G, Falus A, Gay S. Emerging role of extracellular vesicles in inflammatory diseases. Nat Rev. 2014;10:356-64.

29. Xue Y, Wei Z, Ding H, Wang Q, Zhou Z, Zheng S, et al. MicroRNA-19b/221/222 induces endothelial cell dysfunction via suppression of PGC-1alpha in the progression of atherosclerosis. Atherosclerosis. 2015;241:671-81.

30. He L, Thomson JM, Hemann MT, Hernando-Monge E, Mu D, Goodson S, et al. A microRNA polycistron as a potential human oncogene. Nature. 2005;435:828-33.

31. Tsitsiou E, Lindsay MA. microRNAs and the immune response. Curr Opin Pharm. 2009;9:514-20.

32. Liu GL, Yang HJ, Liu B, Liu T. Effects of MicroRNA-19b on the proliferation, apoptosis, and migration of Wilms' tumor cells via the PTEN/PI3K/AKT signaling pathway. J Cell Biochem. 2017;118:3424-34.

33. Nakagawa R, Naka T, Tsutsui H, Fujimoto M, Kimura A, Abe T, et al. SOCS-1 participates in negative regulation of LPS responses. Immunity. 2002;17:677-87.

34. Paracha RZ, Ahmad J, Ali A, Hussain R, Niazi U, Tareen SH, et al. Formal modelling of toll like receptor 4 and JAK/STAT signalling pathways: insight into the roles of SOCS-1, interferonbeta and proinflammatory cytokines in sepsis. PloS ONE. 2014;9: e108466. 